\title{
Amphipathic Helices from Aromatic Amino Acid
}

\section{Oligomers}

\author{
Elizabeth R. Gillies, ${ }^{\dagger}$ Christel Dolain,${ }^{\dagger}$ Jean-Michel Léger,${ }^{*}$ and Ivan Huc ${ }^{\dagger *}$
}

Table of Contents:

1. General procedures and materials.......................................... 2

2. Experimental Procedures for the synthesis of compounds 6 to $17 \ldots \ldots \ldots \ldots . . p g$. 2-9

3. Scheme for the synthesis of amphipathic helices having a larger hydrophilic

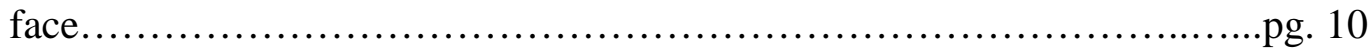

4. Experimental procedures for compounds $\mathbf{1 8}$ to $28 \ldots \ldots \ldots \ldots \ldots \ldots \ldots \ldots \ldots . . \mathrm{pg}$. 10-18

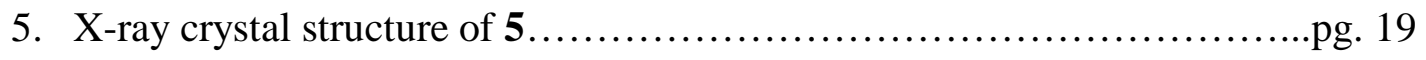

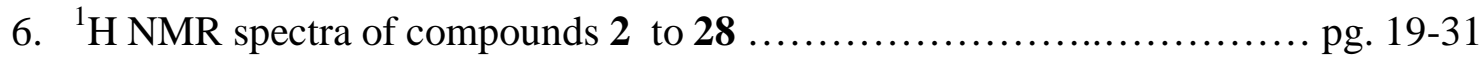


General Procedures and Materials. Unless otherwise noted, materials were obtained from commercial suppliers and used without further purification. THF was distilled from $\mathrm{Na}$ /benzophenone while $\mathrm{CH}_{2} \mathrm{Cl}_{2}$ and diisopropylethylamine (DIEA) were distilled from $\mathrm{CaH}_{2}$ prior to use. ${ }^{1} \mathrm{H} \mathrm{NMR}$ spectra were obtained at $400 \mathrm{MHz}$, and ${ }^{13} \mathrm{C}$ NMR spectra were obtained at $100 \mathrm{MHz}$. Chemical shifts are reported in ppm and are calibrated against residual solvent signals of $\mathrm{CDCl}_{3}(\delta 7.26,77.2)$, DMSO-d $3(\delta 2.50,39.4)$, or $\mathrm{CD}_{3} \mathrm{OD}(\delta$ 3.31, 49.1). All coupling constants are reported in Hz. FT-IR spectroscopic analyses were performed using a solution in $\mathrm{CHCl}_{3}$, a thin film from $\mathrm{CHCl}_{3}$ or a $\mathrm{KBr}$ pellet and are reported in $\mathrm{cm}^{-1}$. Silica gel chromatography was performed using Merck Kieselgel Si 60 . Thin layer chromatography was performed using Merck Kieselgel Si $60 \mathrm{~F}_{254}$ plates. High resolution electrospray ionization time of flight (ESI-TOF) mass spectra were obtained in the positive ion mode and matrix assisted laser desorption ionization time of flight (MALDI-TOF) mass spectra were obtained in positive ion mode using $\alpha$ cyanohydroxycinnamic acid as a matrix.

Dimer amine 6. The same procedure as for the preparation of $\mathbf{3}$ was followed to provide $0.76 \mathrm{~g}$ (99\% yield) of 6 as a yellow solid. IR ( $\mathrm{CHCl}_{3}$ solution): $1720,1608,1552{ }^{1} \mathrm{H}$ NMR (DMSO-d $\left.)_{6}\right): \delta 1.09(\mathrm{~d}, 6 \mathrm{H}, J=6.8), 2.13-2.27(\mathrm{~m}, 3 \mathrm{H}), 3.49-3.53(\mathrm{~m}, 2 \mathrm{H}), 4.09(\mathrm{~s}$, $3 \mathrm{H}), 4.17(\mathrm{~d}, 2 \mathrm{H}, J=6.4), 4.43(\mathrm{t}, 2 \mathrm{H}, J=5.9), 6.34(\mathrm{~s}, 1 \mathrm{H}), 7.09(\mathrm{~d}, 1 \mathrm{H}, J=6.8), 7.36(\mathrm{~d}$, $1 \mathrm{H}, J=8.3), 7.43(\mathrm{t}, 1 \mathrm{H}, J=7.8), 7.67(\mathrm{~s}, 1 \mathrm{H}), 7.70(\mathrm{~s}, 1 \mathrm{H}) 7.78,(\mathrm{t}, 1 \mathrm{H}, J=8.0), 7.94(\mathrm{~d}$, $1 \mathrm{H}, J=8.3), 9.00(\mathrm{~d}, 1 \mathrm{H}, J=7.8), 9.60(\mathrm{t}, 1 \mathrm{H}, J=3.9), 12.37(\mathrm{~s}, 1 \mathrm{H}) .{ }^{13} \mathrm{C}$ NMR (DMSO$\left.\mathrm{d}_{6}\right): \delta 19.8,28.59,28.65,37.2,54.1,67.4,75.2,98.8,102.6,108.0,111.2,116.3,116.8(\mathrm{q}$, $J=288) 117.3,122.3,123.3,129.2,129.7,135.4,136.9,139.2,146.3,147.9,148.0,157.2$ 
$(\mathrm{q}, J=36) 162.9,163.1,163.4,165.8 . \quad \mathrm{MS}$ calcd $[\mathrm{M}+\mathrm{H}]^{+}\left(\mathrm{C}_{30} \mathrm{H}_{31} \mathrm{~N}_{5} \mathrm{O}_{6} \mathrm{~F}_{3}\right): 614.2226$. Found: (TOF MS ES+) 614.2236.

Trimer 7. The same procedure as for the preparation of $\mathbf{5}$ was followed. The product was purified by silica gel chromatography using a gradient from $90 / 10$ to $80 / 20$ toluene/EtOAc to provide $0.62 \mathrm{~g}$ (91\% yield) of 7 as a yellow solid. IR ( $\mathrm{CHCl}_{3}$ solution): 1726, 1677, 1534. ${ }^{1} \mathrm{H}$ NMR $\left(\mathrm{CDCl}_{3}\right): \delta 1.21(\mathrm{~d}, 6 \mathrm{H}, J=6.8), 1.23(\mathrm{~d}, 6 \mathrm{H}, J=6.8), 2.24-2.27(\mathrm{~m}, 2 \mathrm{H})$, 2.34-2.44 (m, 2H), $3.45(\mathrm{~s}, 3 \mathrm{H}), 3.68-3.72(\mathrm{~m}, 2 \mathrm{H}), 4.12(\mathrm{t}, 2 \mathrm{H}, J=5.4), 4.19(\mathrm{~d}, 2 \mathrm{H}, J=$ 6.4), $4.22(\mathrm{~d}, 2 \mathrm{H}, J=6.4), 6.74(\mathrm{~s}, 1 \mathrm{H}), 7.11-7.15(\mathrm{~m}, 1 \mathrm{H}), 7.39(\mathrm{t}, 1 \mathrm{H}, J=7.8), 7.54(\mathrm{~d}$, $1 \mathrm{H}, J=7.3), 7.66-7.73(\mathrm{~m}, 2 \mathrm{H}), 7.78(\mathrm{~d}, 1 \mathrm{H}, J=8.3), 7.85(\mathrm{~s}, 1 \mathrm{H}), 7.93(\mathrm{~s}, 1 \mathrm{H}), 8.08(\mathrm{~d}$, $1 \mathrm{H}, J=8.3), 8.47(\mathrm{~d}, 1 \mathrm{H}, J=8.3), 9.01(\mathrm{~d}, 1 \mathrm{H}, J=7.3), 9.07(\mathrm{~d}, 1 \mathrm{H}, J=7.8), 12.20(\mathrm{~s}, 1 \mathrm{H})$, $12.22(\mathrm{~s}, 1 \mathrm{H}) .{ }^{13} \mathrm{C} \mathrm{NMR}\left(\mathrm{CDCl}_{3}\right): \delta 19.4,28.3,28.4,28.6,37.7,52.5,66.6,75.6,76.0$, $99.3,99.7,100.2,115.5,116.0(\mathrm{q}, J=285), 117.0,118.2,118.4,121.7,122.5,123.5,124.8$, $126.0,127.5,127.8,128.9,134.7,135.7,139.2,140.1,145.6,145.7,150.9,154.3,157.7$ (q, $J=36), 161.8,162.8,163.1,163.4,163.9,164.4$. MS calcd $[\mathrm{M}+\mathrm{H}]^{+}\left(\mathrm{C}_{44} \mathrm{H}_{43} \mathrm{~N}_{7} \mathrm{O}_{10} \mathrm{~F}_{3}\right)$ : 886.3024. Found: (TOF MS ES+) 886.2995.

Pentamer 9. The same procedure as for the preparation of $\mathbf{5}$ was followed. The product was purified by silica gel chromatography using a gradient from $90 / 10$ to $80 / 20$ toluene/EtOAc to provide $0.32 \mathrm{~g}$ ( $87 \%$ yield) of 9 as a yellow solid. IR $\left(\mathrm{CHCl}_{3}\right.$ solution): 3312, 1721, 1677, 1539. ${ }^{1} \mathrm{H}$ NMR $\left(\mathrm{CDCl}_{3}\right): \delta$ 1.22-1.32 (m, 18H), 2.29-2.37 (m, 5H), 2.45-2.53 (m, 2H), $3.22(\mathrm{~s}, 3 \mathrm{H}), 3.68-3.77(\mathrm{~m}, 3 \mathrm{H}), 3.79-3.90(\mathrm{~m}, 1 \mathrm{H}), 3.91-3.95(\mathrm{~m}, 2 \mathrm{H})$, 4.05-4.27 (m, 5H), 4.31-4.39 (m, 3H), $6.51(\mathrm{~s}, 1 \mathrm{H}), 6.79(\mathrm{~s}, 1 \mathrm{H}), 6.87(\mathrm{~s}, 1 \mathrm{H}), 7.07-7.13(\mathrm{~m}$, 
$1 \mathrm{H}), 7.28-7.38(\mathrm{~m}, 3 \mathrm{H}), 7.48(\mathrm{~d}, 3 \mathrm{H}, J=8.3), 7.59-7.65(\mathrm{~m}, 3 \mathrm{H}), 7.76(\mathrm{~d}, 1 \mathrm{H}, J=7.8), 7.83$ $(\mathrm{d}, 1 \mathrm{H}, J=8.3), 8.03(\mathrm{~d}, 1 \mathrm{H}, J=8.3), 8.09(\mathrm{~d}, 1 \mathrm{H}, J=8.3), 8.18(\mathrm{~d}, 1 \mathrm{H}, J=7.8), 8.23(\mathrm{~d}$, $1 \mathrm{H}, J=7.8), 8.34(\mathrm{~d}, 1 \mathrm{H}, J=7.3), 8.45(\mathrm{~d}, 1 \mathrm{H}, J=7.3), 8.53(\mathrm{~d}, 1 \mathrm{H}, J=8.3), 11.50(\mathrm{~s}, 1 \mathrm{H})$, $11.71(\mathrm{~s}, 2 \mathrm{H}), 11.91(\mathrm{~s}, 1 \mathrm{H}) .{ }^{13} \mathrm{C} \mathrm{NMR}\left(\mathrm{CDCl}_{3}\right): \delta 19.5,19.6,19.7,28.0,28.41,28.44$, $28.5,28.6,37.3,37.9,52.4,66.2,66.7,75.5,75.7,76.0,97.5,98.2,99.8,100.2,100.5$, $115.1,115.90(\mathrm{q}, J=287), 115.93(\mathrm{q}, J=287) 116.0,116.76,116.82,116.9,117.0,117.1$, 117.4, 117.6, 121.7, 122.2, 122.7, 124.1, 124.6, 126.2, 126.8, 127.0, 127.7, 128.2, 128.6, 133.5, 133.9, 134.4, 134.6, 138.3, 138.5, 138.8, 139.1, 139.2, 144.8, 145.4, 149.2, 149.6, $150.5,153.7,157.8(\mathrm{q}, J=37), 158.0(\mathrm{q}, J=36) 160.7,161.0,161.6,161.7,161.9,162.9$, 163.2, 163.5, 163.7, 165.0. MS calcd $[\mathrm{M}+\mathrm{H}]^{+}\left(\mathrm{C}_{73} \mathrm{H}_{69} \mathrm{~N}_{12} \mathrm{O}_{15} \mathrm{~F}_{6}\right)$ : 1467.4910. Found: (TOF MS ES+) 1467.4868.

Pentamer amine 10. The same procedure as for the preparation of $\mathbf{3}$ was followed to provide $0.29 \mathrm{~g}$ (99\% yield) of $\mathbf{1 0}$ as a yellow solid. IR $\left(\mathrm{CHCl}_{3}\right.$ solution): 3428,3317 , 1726, 1682, 1543, 1514. ${ }^{1} \mathrm{H}$ NMR $\left(\mathrm{CDCl}_{3}\right): \delta 1.22-1.33$ (m, $\left.18 \mathrm{H}\right), 2.29-2.38(\mathrm{~m}, 5 \mathrm{H})$, 2.41-2.56 (m, 2H), $3.42(\mathrm{~s}, 3 \mathrm{H}), 3.59-3.88(\mathrm{~m}, 6 \mathrm{H}), 3.92(\mathrm{~d}, 2 \mathrm{H}, J=6.4), 4.07-4.16(\mathrm{~m}$, 3H), $4.20(\mathrm{t}, 1 \mathrm{H}, J=7.8), 4.26-4.41(\mathrm{~m}, 4 \mathrm{H}), 5.79(\mathrm{~d}, 1 \mathrm{H}, J=7.3), 6.52(\mathrm{~s}, 1 \mathrm{H}), 6.78(\mathrm{~s}$, 1H), $6.89(\mathrm{~s}, 1 \mathrm{H}), 6.96(\mathrm{t}, 1 \mathrm{H}, J=7.8), 7.07(\mathrm{~m}, 1 \mathrm{H}), 7.26-7.33(\mathrm{~m}, 4 \mathrm{H}), 7.40(\mathrm{~s}, 1 \mathrm{H}), 7.49$ (d, 2H, $J=7.6), 7.65-7.69(\mathrm{~m}, 2 \mathrm{H}), 7.77(\mathrm{~d}, 1 \mathrm{H}, J=8.3), 7.86(\mathrm{~d}, 1 \mathrm{H}, J=8.3), 7.95(\mathrm{~d}, 1 \mathrm{H}$, $J=8.3), 8.04-8.09(\mathrm{~m}, 3 \mathrm{H}), 8.44(\mathrm{~d}, 1 \mathrm{H}, J=7.6), 8.54(\mathrm{~d}, 1 \mathrm{H}, J=7.6), 11.66(\mathrm{~s}, 1 \mathrm{H}), 11.76$ (s, 1H), 11.9 (s, 1H), 11.9 (s, 1H). ${ }^{1} \mathrm{H}$ NMR $\left(\mathrm{CDCl}_{3}\right): \delta$ 19.5, 19.60, 19.63, 19.7, 27.9, $28.4,28.48,28.53,37.5,37.8,52.5,66.5,66.7,75.4,75.7,97.8,98.0,98.9,99.5,100.2$ $109.6,110.1,115.4,115.7,115.96,116.02,116.1(\mathrm{q}, J=288), 117.1,117.27,117.32$ 
$121.4,121.7,122.1,122.5,123.2,127.1,127.2,127.5,128.6,133.6,133.8,133.9,134.5$, $136.5,137.9,138.4,138.5,139.2,143.2,145.1,148.8,148.9,149.9,150.1,157.8(\mathrm{q}, J=$ 37), $157.9(\mathrm{q}, J=37), 161.0,161.2,161.7,161.8,162.0,162.6,163.2,163.4,164.0,164.8$. MS calcd $[\mathrm{M}+\mathrm{H}]^{+}\left(\mathrm{C}_{73} \mathrm{H}_{71} \mathrm{~N}_{12} \mathrm{O}_{13} \mathrm{~F}_{6}\right)$ : 1437.4. Found: (MALDI-TOF) 1437.3.

Dimer acid 11. The same procedure as for the preparation of $\mathbf{8}$ was followed to provide $0.43 \mathrm{~g}$ (95\% yield) of $\mathbf{1 1}$ as a pure yellow solid without chromatographic purification. IR (KBr pellet): $3538,3415,3299,1757,1695,1583,1568,1531,1497 .{ }^{1} \mathrm{H}$ NMR (DMSO$\left.\mathrm{d}_{6}\right): \delta 1.08(\mathrm{~d}, 6 \mathrm{H}, J=6.4), 2.12-2.22(\mathrm{~m}, 3 \mathrm{H}), 3.50(\mathrm{~d}, 2 \mathrm{H}, J=5.1), 4.18(\mathrm{~d}, 2 \mathrm{H}, J=5.4)$, 4.36-4.44 (m, 2H), $7.57(\mathrm{~s}, 1 \mathrm{H}), 7.67(\mathrm{t}, 1 \mathrm{H}, J=8.0), 7.75(\mathrm{~s}, 1 \mathrm{H}), 7.83(\mathrm{t}, 1 \mathrm{H}, J=7.8)$, $7.88(\mathrm{~d}, 1 \mathrm{H}, J=8.0), 8.37$ (d, $1 \mathrm{H}, J=7.3), 8.44(\mathrm{~d}, 1 \mathrm{H}, J=8.3), 8.88$ (d, $1 \mathrm{H}, J=7.3), 9.58$ $9.63(\mathrm{~m}, 1 \mathrm{H}), 11.67$ (s, 1H), 13.5 (br s, 1H). ${ }^{13} \mathrm{C}$ NMR (DMSO-d $)_{6}$ ): $\delta$ 19.7, 28.4, 28.7, $37.2,67.3,76.0,101.0,102.2,116.8(\mathrm{q}, J=288) 117.0,118.3,122.1,123.3,126.4,126.8$, $127.5,128.4,135.1,139.0,139.3,148.4,150.6,154.0,157.2(\mathrm{q}, J=36) 162.7,162.9$, 163.6, 167.5. MS calcd $[\mathrm{M}+\mathrm{H}]^{+}\left(\mathrm{C}_{29} \mathrm{H}_{27} \mathrm{~N}_{5} \mathrm{O}_{8} \mathrm{~F}_{3}\right)$ : 630.1812. Found: (TOF MS ES+) 630.1788.

Heptamer amine 13. The same procedure as for the preparation of $\mathbf{3}$ was followed to provide $0.15 \mathrm{~g}$ (99\% yield) of $\mathbf{1 3}$ as a yellow solid. $\mathrm{IR}\left(\mathrm{CHCl}_{3}\right.$ solution): 1726, 1680, 1547, 1513. ${ }^{1} \mathrm{H}$ NMR $\left(\mathrm{CDCl}_{3}\right): \delta 1.13-1.37(\mathrm{~m}, 24 \mathrm{H}), 2.17-2.55(\mathrm{~m}, 10 \mathrm{H}), 3.07(\mathrm{~s}, 3 \mathrm{H})$, 3.16-3.36 (m, 1H), 3.48-3.62 (m, 2H), 3.70-4.10 (m, 15H), 4.12-4.42 (m, 2H), 4.50-4.58 (m, 1H), $5.73(\mathrm{~d}, 1 \mathrm{H}, J=6.8), 6.39(\mathrm{~s}, 1 \mathrm{H}), 6.49(\mathrm{~s}, 1 \mathrm{H}), 6.54(\mathrm{~s}, 2 \mathrm{H}), 6.73(\mathrm{~s}, 1 \mathrm{H}), 6.85-$ $6.91(\mathrm{~m}, 3 \mathrm{H}), 7.13-7.26(\mathrm{~m}, 4 \mathrm{H}), 7.31-7.39(\mathrm{~m}, 2 \mathrm{H}), 7.55-7.60(\mathrm{~m}, 1 \mathrm{H})$, 7.60-7.68 (m, 3H), 
$7.80(\mathrm{~d}, 1 \mathrm{H}, J=8.1), 7.84-7.86(\mathrm{~m}, 2 \mathrm{H}), 7.96-8.10(\mathrm{~m}, 4 \mathrm{H}), 8.16(\mathrm{~d}, 1 \mathrm{H}, J=7.3), 8.20(\mathrm{~d}$, $1 \mathrm{H}, J=7.6), 11.02(\mathrm{~s}, 1 \mathrm{H}), 11.25(\mathrm{~s}, 1 \mathrm{H}), 11.28(\mathrm{~s}, 1 \mathrm{H}), 11.37(\mathrm{~s}, 1 \mathrm{H}), 11.50(\mathrm{~s}, 1 \mathrm{H}), 11.68$ (s, 1H). ${ }^{13} \mathrm{C} \mathrm{NMR}\left(\mathrm{CDCl}_{3}\right): \delta 19.38,19.44,19.5,19.55,19.63,19.69,19.72,27.9,28.2$ $28.3,28.47,28.55,28.9,29.9,37.4,37.6,39.7,52.3,65.8,66.6,68.6,75.16,75.21,75.6$, 97.6, 97.7, 98.4, 98.5, 98.9, 99.1, 100.1, 109.6, 110.1, 115.4, 115.6, 115.9, 116.0, 116.17 $(\mathrm{q}, J=288), 116.18(\mathrm{q}, J=288), 116.20,116.3,116.5(\mathrm{q}, J=288), 116.6,116.8,117.0$, 117.2, 121.4, 121.5, 121.8, 122.0, 122.2, 122.3, 122.9, 126.4, 126.5, 126.9, 127.1, 127.4, 128.4, 132.9, 133.1, 133.4, 133.65, 133.72, 134.1, 136.1, 137.6, 137.8, 138.1, 138.3, 138.8, $143.1,144.7,148.0,148.4,148.7,149.6,149.7,149.77,149.84,157.7$ (q, $J=36), 157.9$ (q, $J=36), 159.3,160.1,160.8,161.2,161.47,161.55,162.6,162.68,162.74,162.8,163.0$ 163.1, 163.2, 164.6. MS calcd $[\mathrm{M}+\mathrm{H}]^{+}\left(\mathrm{C}_{102} \mathrm{H}_{97} \mathrm{~N}_{17} \mathrm{O}_{18} \mathrm{~F}_{9}\right)$ : 2018.7054. Found: (TOF MS ES+) 2018.7186 .

Decamer 14. The same procedure as for the preparation of 5 was followed. The product was purified by silica gel chromatography using a gradient from $80 / 20$ to $50 / 50$ toluene/EtOAc to provide $87 \mathrm{mg}$ (74\% yield) of $\mathbf{1 4}$ as a yellow solid. IR $\left(\mathrm{CHCl}_{3}\right.$ solution): $3323,3161,1774,1719,1682,1499 .{ }^{1} \mathrm{H}$ NMR $\left(\mathrm{CDCl}_{3}\right): \delta 1.12-1.36(\mathrm{~m}, 36 \mathrm{H}), 2.15-2.50$ (m, 14H), 3.81-3.63 (m, 4H), 3.73-4.11 (m, 20H), 4.19-4.32 (m, 4H), $6.03(\mathrm{~s}, 1 \mathrm{H}), 6.19(\mathrm{~s}$, 1H), $6.22(\mathrm{~s}, 1 \mathrm{H}), 6.28(\mathrm{~s}, 1 \mathrm{H}), 6.31(\mathrm{~s}, 1 \mathrm{H}), 6.32(\mathrm{~s}, 1 \mathrm{H}), 6.44(\mathrm{~s}, 1 \mathrm{H}), 6.56(\mathrm{~s}, 1 \mathrm{H}), 6.93-$ $6.97(\mathrm{~m}, 2 \mathrm{H}), 7.00(\mathrm{~s}, 1 \mathrm{H}), 7.04(\mathrm{t}, 1 \mathrm{H}, J=5.4), 7.07-7.42(\mathrm{~m}, 16 \mathrm{H}), 7.53-7.56(\mathrm{~m}, 3 \mathrm{H})$, $7.67(\mathrm{~d}, 1 \mathrm{H}, J=8.1), 7.76-7.83(\mathrm{~m}, 3 \mathrm{H}), 7.89(\mathrm{~d}, 1 \mathrm{H}, J=8.1), 7.93(\mathrm{~d}, 1 \mathrm{H}, J=8.1), 7.97-$ $8.06(\mathrm{~m}, 5 \mathrm{H}), 8.11(\mathrm{t}, 1 \mathrm{H}, J=5.4), 8.28(\mathrm{~d}, 1 \mathrm{H}, J=7.8), 10.64(\mathrm{~s}, 1 \mathrm{H}), 10.68(\mathrm{~s}, 1 \mathrm{H}), 10.71$ (s, 1H), $10.81(\mathrm{~s}, 1 \mathrm{H}), 10.82(\mathrm{~s}, 1 \mathrm{H}), 10.90(\mathrm{~s}, 1 \mathrm{H}), 10.98(\mathrm{~s}, 1 \mathrm{H}), 11.14(\mathrm{~s}, 1 \mathrm{H}), 11.32(\mathrm{~s}$, 
1H). ${ }^{13} \mathrm{C} \mathrm{NMR}\left(\mathrm{CDCl}_{3}\right): \delta 19.3,19.40,19.44,19.47,19.50,19.52,19.54,19.6,19.71$, $19.74,19.78,27.9,27.97,28.05,28.16,28.23,28.36,28.42,28.7,29.9,37.2,38.6,38.7$, 39.2, 52.2, 65.9, 66.2, 67.1, 67.9, 75.1, 75.4, 75.7, 76.8, 77.1, 97.46, 97.52, 97.6, 98.0, 98.4, $98.69,98.71,99.5,99.89,99.92,115.4,115.5,115.6,115.77,115.80,116.08,116.09$ (q, $J$ $=288), 116.13(\mathrm{q}, J=288), 116.36(\mathrm{q}, J=288), 116.36,116.42,116.49(\mathrm{q}, J=288), 116.6$, 116.7, 116.8, 117.0, 117.37, 117.41, 121.2, 121.3, 121.6, 121.9, 121.97, 122.02, 122.1, $122.35,122.40,123.7,124.1,125.3,125.5,126.0,126.2,126.5,127.0,127.2,128.2,128.3$, $132.1,132.46,132.49,132.6,132.8,132.9,133.7,133.8,134.1,137.30,137.33,137.4$, $137.8,137.9,138.2,138.6,138.7,144.5,144.7,148.0,148.2,148.6,148.76,148.80,149.0$ 149.1, 149.7, 153.0, $157.8(\mathrm{q}, J=36), 157.92(\mathrm{q}, J=37), 157.94(\mathrm{q}, J=37), 159.0,159.1$, $159.4,159.6,159.7,160.1,160.5,160.6,160.9,161.4,161.9,162.1,162.3,162.6,162.7$, 162.8, 162.88, 162.91, 163.0, 164.5. MS calcd $[\mathrm{M}+\mathrm{H}]^{+}\left(\mathrm{C}_{145} \mathrm{H}_{135} \mathrm{~N}_{24} \mathrm{O}_{27} \mathrm{~F}_{12}\right): 2871.9737$. Found: (TOF MS ES+) 2871.9800.

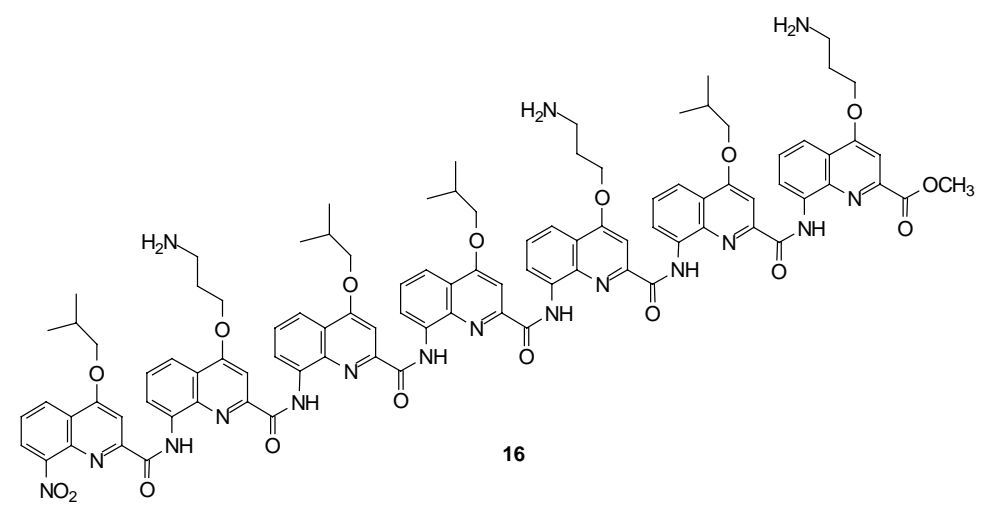

Deprotected heptamer 16. The same procedure as for the preparation of $\mathbf{1 5}$ was followed to provide $39 \mathrm{mg}$ (91\% yield) of $\mathbf{1 6}$ as a yellow solid. IR (thin film): 3319, 1677, 1537. ${ }^{1} \mathrm{H}$ NMR $\left(\mathrm{CDCl}_{3}\right): \delta 1.17-1.35(\mathrm{~m}, 24 \mathrm{H}), 2.04-2.09(\mathrm{~m}, 2 \mathrm{H}), 2.23-2.52(\mathrm{~m}, 8 \mathrm{H}), 3.00-3.19$ (m, 9H), 3.83-4.16 (m, 10H), 4.30-4.38 (m, 3H), 4.44-4.49 (m, 1H), $6.49(\mathrm{~s}, 2 \mathrm{H}), 6.53(\mathrm{~s}$ 1H), $6.62(\mathrm{~s}, 1 \mathrm{H}), 6.82(\mathrm{~s}, 1 \mathrm{H}), 7.09(\mathrm{~s}, 1 \mathrm{H}), 7.12(\mathrm{~s}, 1 \mathrm{H}), 7.22-7.28(\mathrm{~m}, 5 \mathrm{H}), 7.40-7.45(\mathrm{~m}$ 
4H), $7.53(\mathrm{~d}, 1 \mathrm{H}, J=7.3), 7.57(\mathrm{~d}, 1 \mathrm{H}, J=7.6), 7.74(\mathrm{~d}, 1 \mathrm{H}, J=8.3), 7.84-7.92(\mathrm{~m}, 3 \mathrm{H})$, $8.02-8.20(\mathrm{~m}, 5 \mathrm{H}), 8.27(\mathrm{~d}, 1 \mathrm{H}, J=7.3), 8.39(\mathrm{~d}, 1 \mathrm{H}, J=8.1), 11.14(\mathrm{~s}, 1 \mathrm{H}), 11.18(\mathrm{~s}, 1 \mathrm{H})$, $11.19(\mathrm{~s}, 1 \mathrm{H}), 11.36(\mathrm{~s}, 1 \mathrm{H}), 11.47(\mathrm{~s}, 1 \mathrm{H}), 11.63(\mathrm{~s}, 1 \mathrm{H}) .{ }^{13} \mathrm{C} \mathrm{NMR}\left(\mathrm{CDCl}_{3}\right): \delta 19.35$ $19.40,19.5,19.6,19.70,19.72,28.2,28.28,28.33,29.8,32.7,32.8,39.1,39.2,39.3,52.2$, $66.76,66.84,67.1,75.1,75.4,75.5,75.7,97.7,97.8,98.4,98.9,99.5,100.0,100.3,115.9$, 116.0, 116.1, 116.2, 116.4, 116.5, 116.6, 116.85, 116.92, 117.3, 121.5, 121.7, 122.4, 122.5, $122.7,123.8,124.0,125.8,125.9,126.3,126.6,126.8,127.1,127.8,128.1,132.9,133.6$, $133.77,133.81,134.3,137.7,137.9,138.2,138.5,138.9,144.9,145.2,148.86,148.94$ $149.2,150.1,153.2,159.5,160.1,160.3,160.7,160.9,161.3,161.9,162.7,162.8,162.89$ 162.94, 163.0, 163.9. MS calcd $[\mathrm{M}+\mathrm{H}]^{+}\left(\mathrm{C}_{96} \mathrm{H}_{98} \mathrm{~N}_{17} \mathrm{O}_{17}\right)$ : 1760.7327. Found: (TOF MS ES+) 1760.7216 .

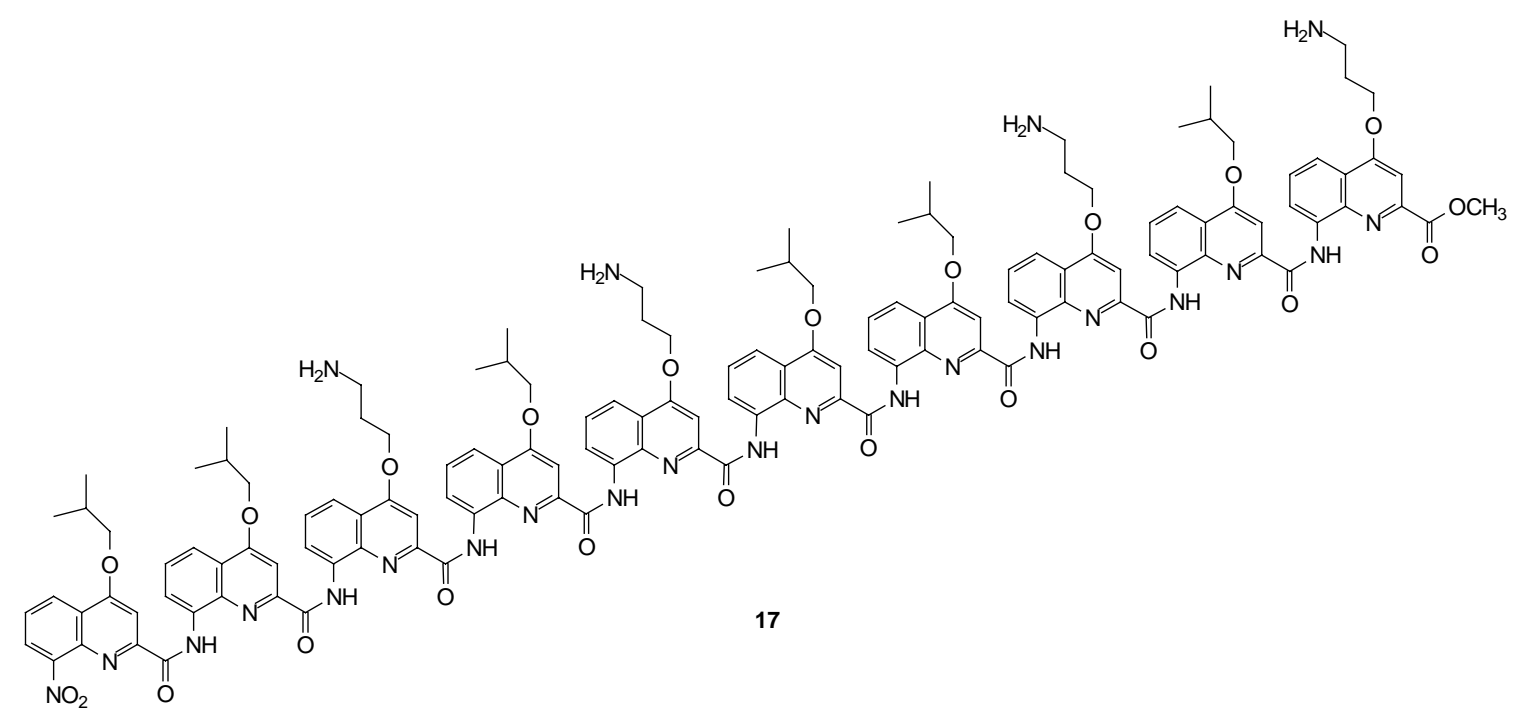

Deprotected decamer 17. The same procedure as for the preparation of $\mathbf{1 5}$ was followed to provide $71 \mathrm{mg}$ (95\% yield) of $\mathbf{1 7}$ as a yellow solid. IR (thin film): 3310, 1683, 1534. ${ }^{1} \mathrm{H}$ NMR $\left(\mathrm{CDCl}_{3}\right): \quad \delta 1.25-1.33(\mathrm{~m}, 36 \mathrm{H}), 2.06-2.46(\mathrm{~m}, 14 \mathrm{H}), 2.93-3.16(\mathrm{~m}, 11 \mathrm{H}), 3.74-$ $4.21(\mathrm{~m}, 20 \mathrm{H}), 6.09(\mathrm{~s}, 1 \mathrm{H}), 6.12(\mathrm{~s}, 1 \mathrm{H}), 6.14(\mathrm{~s}, 1 \mathrm{H}), 6.32(\mathrm{~s}, 1 \mathrm{H}), 6.33(\mathrm{~s}, 1 \mathrm{H}), 6.34(\mathrm{~s}$, 
1H), $6.48(\mathrm{~s}, 1 \mathrm{H}), 6.58(\mathrm{~s}, 1 \mathrm{H}), 6.94-7.38(\mathrm{~m}, 17 \mathrm{H}), 7.46(\mathrm{~d}, 1 \mathrm{H}, J=7.3) 7.63(\mathrm{t}, 2 \mathrm{H}, J$ 7.9), $7.73(\mathrm{~d}, 1 \mathrm{H}, J=8.3), 7.77(\mathrm{~d}, 2 \mathrm{H}, J=8.1), 7.90-8.00(\mathrm{~m}, 6 \mathrm{H}), 8.05(\mathrm{t}, 2 \mathrm{H}, J=7.2), 8.27(\mathrm{~d}$, 1H), $10.66(\mathrm{~s}, 3 \mathrm{H}), 10.68(\mathrm{~s}, 1 \mathrm{H}), 10.77(\mathrm{~s}, 1 \mathrm{H}), 10.88(\mathrm{~s}, 1 \mathrm{H}), 10.93(\mathrm{~s}, 1 \mathrm{H}), 11.22(\mathrm{~s}, 1 \mathrm{H})$ $11.32(\mathrm{~s}, 1 \mathrm{H}) .{ }^{13} \mathrm{C} \mathrm{NMR}\left(\mathrm{CDCl}_{3}\right): \delta 19.3,19.40,19.43,19.46,19.48,19.54,19.7,19.7$, $19.8,28.18,28.23,28.3,29.9,31.6,32.1,32.47,32.51,39.0,39.1,39.2,52.1,66.5,66.6$, $66.9,75.1,75.3,75.6,97.4,97.6,98.1,98.4,98.5,98.7,98.8,99.6,99.9,100.1,115.7$, 115.8, 115.9, 116.0, 116.16, 116.24, 116.6, 116.7, 116.76, 116.81, 117.3, 121.3, 121.4, $121.6,122.0,122.1,122.2,122.3,123.6,124.0,125.4,125.8,125.9,126.3,126.8,127.0$, $127.8,128.1,132.56,132.64,132.8,133.2,133.61,133.65,134.1,137.3,137.4,137.7$, 137.96, 138.01, 138.7, 133.6, 144.9, 148.3, 148.4, 148.6, 148.7, 148.78, 148.85, 148.9, $149.8,153.0,159.08,159.13,159.2,159.3,159.6,160.1,160.5,160.8,161.0,161.8$ $162.20,162.30,162.5,162.5,162.65,162.74,162.9$. MS calcd $[\mathrm{M}+\mathrm{H}]^{+}\left(\mathrm{C}_{137} \mathrm{H}_{139} \mathrm{~N}_{24} \mathrm{O}_{23}\right)$ : 2488.0446. Found: (TOF MS ES+) 2488.0493. 

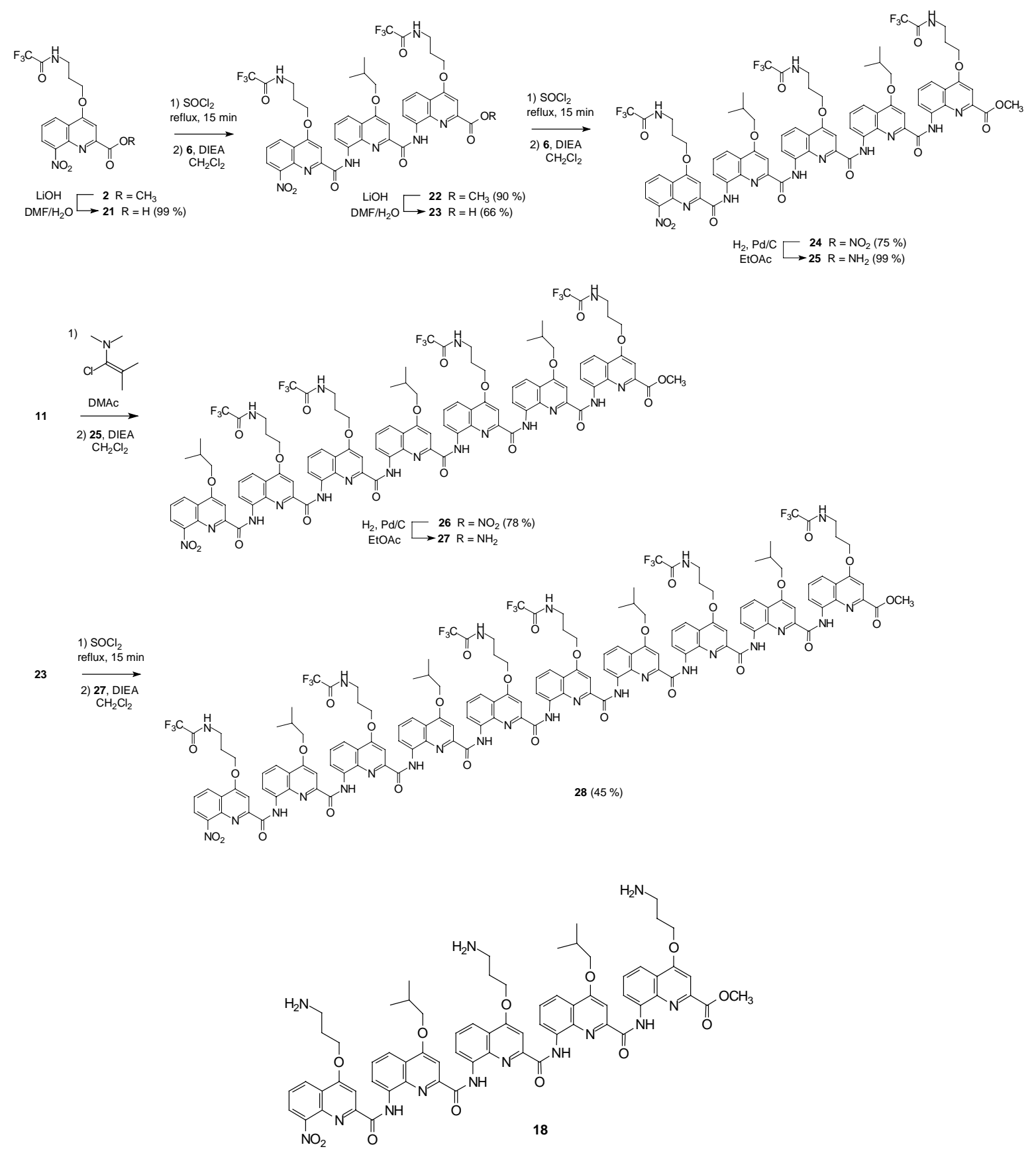

Deprotected pentamer 18. The same procedure as for the preparation of $\mathbf{1 5}$ was followed to provide $41 \mathrm{mg}$ (99\% yield) of $\mathbf{1 8}$ as a yellow solid. IR (thin film): 3306, 1683, 1537. ${ }^{1} \mathrm{H}$ NMR $\left(\mathrm{CDCl}_{3}\right): \delta$ 1.21-1.31 (m, 12H), 2.10-2.18 (m, 4H), 2.26-2.38 (m, 3H), 2.48-2.55 (m, 1H), 3.06-3.20 (m, 9H), 3.88-3.96 (m, 2H), 4.12-4.30 (m, 5H), 4.35-4.39 (m, 1H), 4.52$4.57(\mathrm{~m}, 1 \mathrm{H}), 4.69-4.23(\mathrm{~m}, 1 \mathrm{H}), 6.60(\mathrm{~s}, 1 \mathrm{H}), 6.80(\mathrm{~s}, 1 \mathrm{H}), 6.82(\mathrm{~s}, 1 \mathrm{H}), 7.29-7.39(\mathrm{~m}, 3 \mathrm{H})$, 
7.44-7.48 (m, 2H), $7.50(\mathrm{~s}, 1 \mathrm{H}), 7.64(\mathrm{t}, 2 \mathrm{H}, J=7.8), 7.80(\mathrm{~d}, 1 \mathrm{H}, J=8.3), 7.94(\mathrm{~d}, 1 \mathrm{H}, J=$ 8.1), $8.01(\mathrm{~d}, 1 \mathrm{H}, J=8.1), 8.07(\mathrm{~d}, 1 \mathrm{H}, J=8.1), 8.15(\mathrm{~d}, 2 \mathrm{H}, J=7.1), 8.46-8.52(\mathrm{~m}, 3 \mathrm{H})$, $11.53(\mathrm{~s}, 1 \mathrm{H}), 11.73(\mathrm{~s}, 2 \mathrm{H}), 11.94(\mathrm{~s}, 1 \mathrm{H}) .{ }^{1} \mathrm{H}$ NMR $\left(\mathrm{CDCl}_{3}\right): \delta 19.4,19.5,19.6,28.3$, $28.4,32.5,32.6,39.07,39.12,39.2,52.2,66.8,67.0,67.5,75.3,75.6,97.3,98.1,99.7$, $100.4,115.4,116.37,116.43,116.5,116.7,117.0,117.2,117.3,121.7,121.8,122.1,122.5$, $123.9,124.5,125.9,126.7,126.9,127.5,127.86,127.89,133.66,133.70,134.1,134.4$, $138.1,138.4,138.5,139.0,139.1,145.3,149.15,149.22,150.6,153.7,160.6,160.9,161.3$ 162.0, 162.2, 162.97, 162.99, 163.2, 163.5, 164.0. MS calcd $[\mathrm{M}+\mathrm{H}]^{+}\left(\mathrm{C}_{68} \mathrm{H}_{69} \mathrm{~N}_{13} \mathrm{O}_{13}\right)$ : 1275.5138. Found: (TOF MS ES+) 1275.5242.

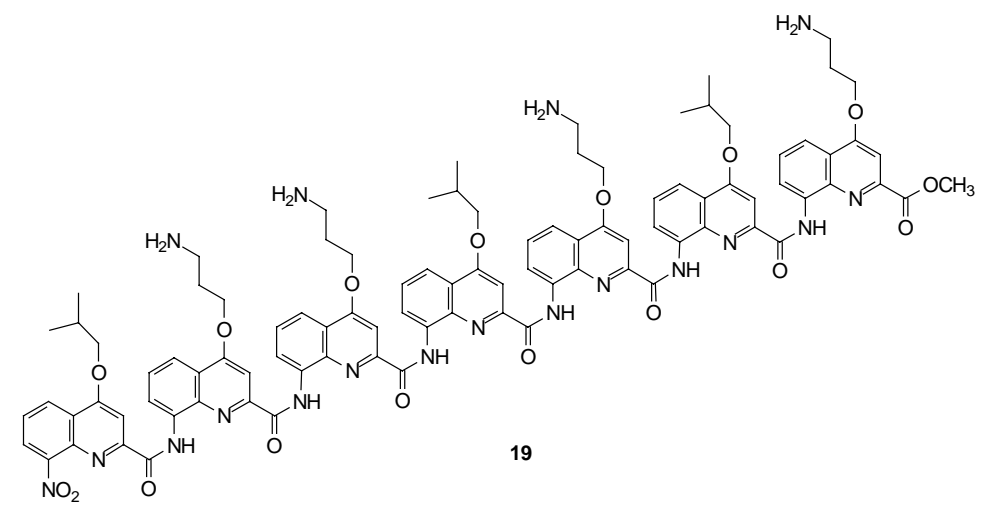

Deprotected heptamer 19. The same procedure as for the preparation of $\mathbf{1 5}$ was followed to provide $32 \mathrm{mg}$ (94\% yield) of $\mathbf{1 9}$ as a yellow solid. IR (thin film): 3306, 1683, 1543. ${ }^{1} \mathrm{H}$ NMR $\left(\mathrm{CDCl}_{3}\right): \delta 1.18-1.25(\mathrm{~m}, 18 \mathrm{H}), 2.19-2.52(\mathrm{~m}, 11 \mathrm{H}), 3.02-3.25(\mathrm{~m}, 11 \mathrm{H}), 3.84(\mathrm{~d}$, $2 \mathrm{H}, J=5.6), 3.95-4.00(\mathrm{~m}, 2 \mathrm{H}), 4.05-4.16(\mathrm{~m}, 4 \mathrm{H}), 4.26-4.35(\mathrm{~m}, 4 \mathrm{H}), 4.47-4.51(\mathrm{~m}, 2 \mathrm{H})$, $6.49(\mathrm{~s}, 1 \mathrm{H}), 6.51(\mathrm{~s}, 1 \mathrm{H}), 6.56(\mathrm{~s}, 1 \mathrm{H}), 6.62(\mathrm{~s}, 1 \mathrm{H}), 6.78(\mathrm{~s}, 1 \mathrm{H}), 7.10(\mathrm{~s}, 1 \mathrm{H}), 7.16(\mathrm{~s}, 1 \mathrm{H})$ 7.21-7.32 (m, 4H), 7.40-7.48 (m, 4H), $7.52(\mathrm{~d}, 2 \mathrm{H}, J=4.6), 7.75(\mathrm{~d}, 1 \mathrm{H}, J=8.1), 7.78-$ 7.91(m, 3H), $7.99(\mathrm{~d}, 1 \mathrm{H}, J=7.8), 8.09(\mathrm{t}, 2 \mathrm{H}, J=8.8), 8.17(\mathrm{t}, 2 \mathrm{H}, J=8.0), 8.31(\mathrm{~d}, 1 \mathrm{H}, J$ = 7.6), $8.40(\mathrm{~d}, 1 \mathrm{H}, J=7.6), 11.16(\mathrm{~s}, 1 \mathrm{H}), 11.18(\mathrm{~s}, 1 \mathrm{H}), 11.22(\mathrm{~s}, 1 \mathrm{H}), 11.36(\mathrm{~s}, 1 \mathrm{H})$ $11.47(\mathrm{~s}, 1 \mathrm{H}), 11.64(\mathrm{~s}, 1 \mathrm{H}) .{ }^{13} \mathrm{C} \mathrm{NMR}\left(\mathrm{CDCl}_{3}\right): \delta 19.38,19.45,19.56,19.63,19.76,28.2$ 
$28.3,28.4,28.5,32.73,39.2,39.26,39.35,52.2,66.8,66.9,67.0,67.1,75.2,75.5,75.7$, 97.6, 97.8, 98.5, 98.8, 99.5, 100.0, 100.3, 115.89, 115.94, 116.0, 116.2, 116.4, 116.5, 116.6, $116.8,116.9,117.2,121.5,121.72,121.74,122.4,122.6,123.8,124.2,125.8,126.1,126.1$, $126.6,127.0,127.2,127.9,128.2,128.96,129.02,132.86,132.94,133.6,133.8,133.9$, $134.3,137.7,138.0,138.2,138.5,138.9,144.9,145.2,148.9,149.0,149.16,149.21,150.1$, $153.2,159.5,160.1,160.3,160.7,161.0,161.4,162.0,162.8,162.86,162.90,163.0,163.1$, 163.9. MS calcd $[\mathrm{M}+\mathrm{H}]^{+}\left(\mathrm{C}_{95} \mathrm{H}_{97} \mathrm{~N}_{18} \mathrm{O}_{17}\right)$ : 1761.7279. Found: (TOF MS ES+) 1761.7208.

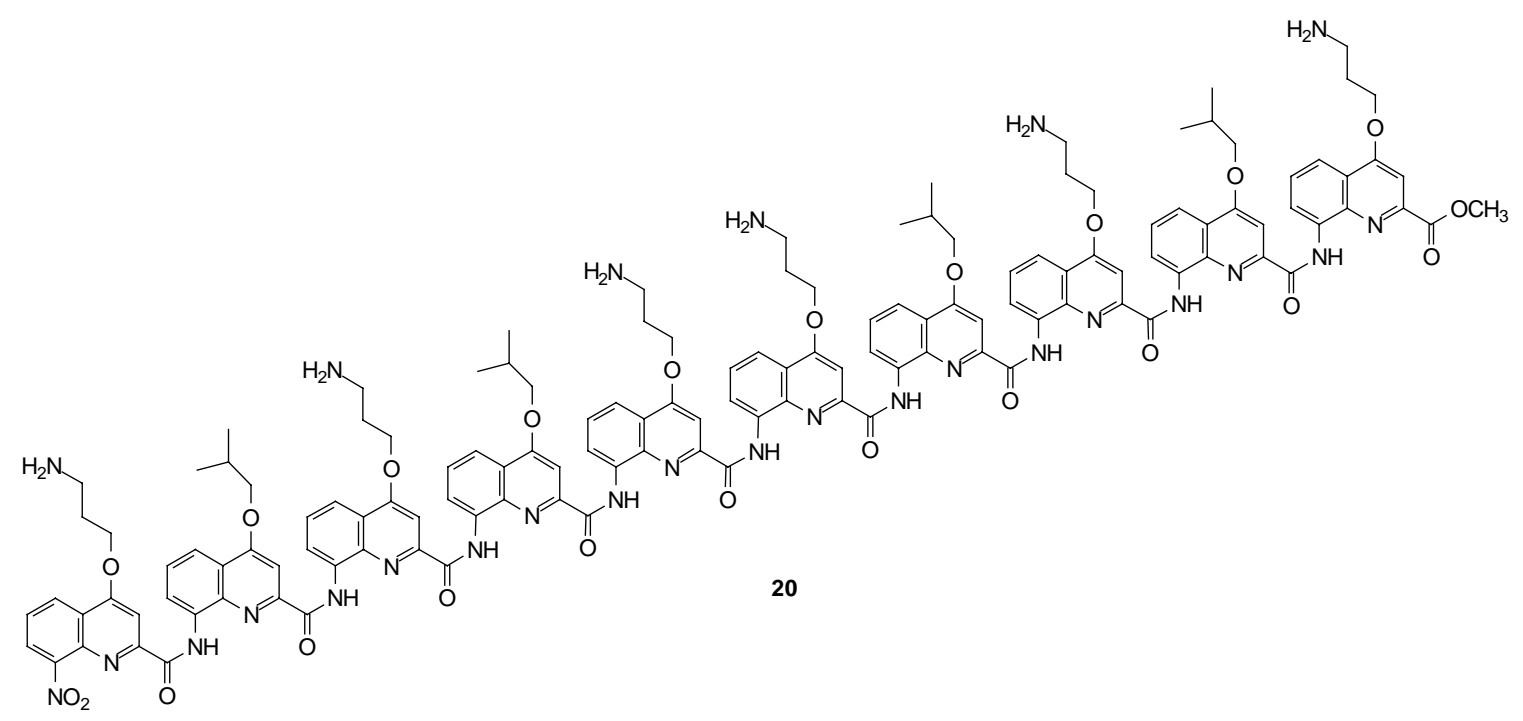

Deprotected decamer 20. The same procedure as for the preparation of $\mathbf{1 5}$ was followed to provide $20 \mathrm{mg}$ (99\% yield) of $\mathbf{2 0}$ as a yellow solid. IR (thin film): 3306, 1683, 1543. ${ }^{1} \mathrm{H}$ $\operatorname{NMR}\left(\mathrm{CDCl}_{3}\right) \delta 2.24-2.44(\mathrm{~m}, 16 \mathrm{H}), 2.92-3.26(\mathrm{~m}, 15 \mathrm{H}), 3.72-4.42(\mathrm{~m}, 20 \mathrm{H}), 6.05(\mathrm{~s}, 1 \mathrm{H})$, $6.14(\mathrm{~s}, 2 \mathrm{H}), 6.35(\mathrm{~s}, 2 \mathrm{H}), 6.40(\mathrm{~s}, 1 \mathrm{H}), 6.48(\mathrm{~s}, 1 \mathrm{H}), 6.56(\mathrm{~s}, 1 \mathrm{H}), 6.95-8.12(\mathrm{~m}, 39 \mathrm{H}), 8.25$ (d, 1H, J = 7.6), $10.61(\mathrm{~s}, 1 \mathrm{H}), 10.64(\mathrm{~s}, 1 \mathrm{H}), 10.69(\mathrm{~s}, 1 \mathrm{H}), 10.72(\mathrm{~s}, 1 \mathrm{H}), 10.80(\mathrm{~s}, 1 \mathrm{H})$ 10.93 (s, 1H), 10.99 (s, 1H), 11.22 (s, 1H), 11.33 (s, 1H). ${ }^{13} \mathrm{C}$ NMR (4/1 $\left.\mathrm{CDCl}_{3} / \mathrm{CD}_{3} \mathrm{OD}\right): \delta$ $18.9,19.05,19.08,19.12,19.14,19.2,19.3,28.0,28.1,31.8,38.3,38.4,38.5,38.6,38.7$ $38.8,51.8,66.3,66.5,66.7,66.9,74.9,75.0,75.1,97.1,97.4,97.5,97.9,98.37,98.44,99.1$, 
99.2, 99.6, 99.9, 115.4, 115.5, 115.7, 115.8, 115.9, 116.4, 116.5, 116.6, 116.7, 117.4, 120.9, $121.1,121.5,121.65,121.71,121.8,122.0,122.1,123.2,125.4,125.5,125.8,125.9,126.2$, $126.8,127.7,131.96,132.05,132.2,132.5,132.7,132.9,133.2,133.7,137.1,137.4$, $137.68,137.7,138.35,138.43,144.4,144.7,147.9,148.2,148.3,148.5,148.6,149.5$, $152.7,158.9,159.0,159.07,159.14,159.5,160.0,160.5,160.8,160.9,161.6,162.1,162.3$, $162.4,162.6,162.7,162.8,163.0,163.6,167.0$. MS calcd $[\mathrm{M}+\mathrm{H}]^{+}\left(\mathrm{C}_{135} \mathrm{H}_{137} \mathrm{~N}_{26} \mathrm{O}_{23}\right)$ : 2490.0350. Found: (TOF MS ES+) 2490.0337.

Monomer acid 21. The monomer 2 (1.0 g, $2.5 \mathrm{mmol}, 1.0$ equiv.) was dissolved in DMF $(50 \mathrm{~mL})$ and the solution was cooled to $0{ }^{\circ} \mathrm{C}$. $\mathrm{LiOH} \cdot \mathrm{H}_{2} \mathrm{O}(0.21 \mathrm{~g}, 5.0 \mathrm{mmol}, 2.0$ equiv. $)$ in $\mathrm{H}_{2} \mathrm{O}(4.9 \mathrm{~mL})$ was added and the reaction mixture was stirred at $0{ }^{\circ} \mathrm{C}$ overnight. The reaction mixture was acidified with $1 \mathrm{M} \mathrm{HCl}$, then diluted with $\mathrm{H}_{2} \mathrm{O}$ at rt. The resulting solution was extracted with $2 / 1 \mathrm{CHCl}_{3} / \mathrm{iPrOH}$. The combined organic layers were dried with $\mathrm{MgSO}_{4}$ and evaporated. The resulting oil was azeotroped with toluene providing 0.96 $\mathrm{g}$ (99 \% yield) of $\mathbf{2 1}$ as a yellow solid. IR (KBr pellet): 3402, 3238, 1739, 1724, 1588, 1562, 1535. ${ }^{1} \mathrm{H}$ NMR (DMSO-d $\left.{ }_{6}\right): \delta 2.11-2.17$ (m, 2H), 3.47-3.52 (m, 2H), $4.44(\mathrm{t}, 2 \mathrm{H}, J$ $=5.9), 7.67(\mathrm{~s}, 1 \mathrm{H}), 7.83(\mathrm{t}, 1 \mathrm{H}, J=7.9), 8.34(\mathrm{~d}, 1 \mathrm{H}, J=7.1), 8.46(\mathrm{~d}, 1 \mathrm{H}, J=8.1), 9.55-$ $9.59(\mathrm{~m}, 1 \mathrm{H}), 13.76(\mathrm{br} \mathrm{s}, 1 \mathrm{H}) .{ }^{13} \mathrm{C}$ NMR (DMSO-d 6 ): $\delta$ 28.6, 37.2, 67.8, 103.5, 116.9 (q, $J=288), 123.1,125.2,126.4,127.6,139.7,149.3,153.3,157.3(\mathrm{q}, J=36), 163.0,166.9$. MS calcd $[\mathrm{M}+\mathrm{H}]^{+}\left(\mathrm{C}_{15} \mathrm{H}_{13} \mathrm{~N}_{3} \mathrm{O}_{6} \mathrm{~F}_{3}\right): 388.0756$. Found: (TOF MS ES+) 388.0749.

Trimer 22. The same procedure as for the preparation of $\mathbf{5}$ was followed except that the resulting acid chloride was dissolved in DMAc prior to being added to the amine via 
cannula. The product was purified by silica gel chromatography using a gradient from 90/10 to 60/40 toluene/EtOAc to provide $0.79 \mathrm{~g}$ (90\% yield) of 22 as a yellow solid. IR (KBr pellet): 3386, 3310, 1731, 1683, 1531. ${ }^{1} \mathrm{H}$ NMR (DMSO-d 6$): \delta 1.15(\mathrm{~d}, 6 \mathrm{H}, J=6.6)$, 2.09-2.18 (m, 2H), 2.22-2.34 (m, 3H), 3.40 (s, 3H), 3.48-3.61 (m, 4H), $4.16(\mathrm{t}, 2 \mathrm{H}, J=5.3)$, $4.29(\mathrm{~d}, 2 \mathrm{H}, J=6.4), 4.59(\mathrm{t}, 2 \mathrm{H}, J=5.4), 6.72(\mathrm{~s}, 1 \mathrm{H}), 7.59(\mathrm{t}, 1 \mathrm{H}, J=7.8), 7.69(\mathrm{~d}, 1 \mathrm{H}, J$ $=7.3), 7.77(\mathrm{t}, 1 \mathrm{H}, J=8.0), 7.82-7.91(\mathrm{~m}, 4 \mathrm{H}), 8.05(\mathrm{~d}, 1 \mathrm{H}, J=8.3), 8.48(\mathrm{~d}, 1 \mathrm{H}, J=8.1)$, $8.89(\mathrm{~d}, 1 \mathrm{H}, J=7.3), 8.99(\mathrm{~d}, 1 \mathrm{H}, J=7.8), 9.62(\mathrm{br} \mathrm{s}, 2 \mathrm{H}), 11.97(\mathrm{~s}, 1 \mathrm{H}), 12.07$ (s, $1 \mathrm{H}) .{ }^{13} \mathrm{C}$ NMR: $\delta$ 18.8, 27.7, 28.0, 28.1, 36.6, 36.7, 52.1, 66.2, 66.7, 75.2, 98.8, 99.1, 99.9, 115.7, $115.9(\mathrm{q}, J=288), 116.9,117.56,117.60,118.0,118.1,121.4,122.1,122.8,124.9,125.9$, 127.1, 127.4, 128.4, 133.8, 133.9, 134.9, 135.0, 138.7, 138.75, 138.78, 139.5, 139.6, 145.0, $145.1,150.4,153.5,157.96(\mathrm{q}, J=37), 157.98(\mathrm{q}, J=37), 161.7,162.4,162.5,162.89$, 162.94, 163.7, 164.4, MS calcd $[\mathrm{M}+\mathrm{H}]^{+}\left(\mathrm{C}_{45} \mathrm{H}_{40} \mathrm{~N}_{8} \mathrm{O}_{11} \mathrm{~F}_{6}\right)$ : 1005.2618. Found: (TOF MS ES+) 1005.2603.

Trimer acid 23. The same procedure was followed as for the preparation of $\mathbf{8}$ except that following acidification with $1 \mathrm{M} \mathrm{HCl}$ and dilution with water, the product was extracted into 2/1 $\mathrm{CHCl}_{3} / \mathrm{iPrOH}$. The combined organic layers were dried with $\mathrm{MgSO}_{4}$ and evaporated, then the product was purified by silica gel chromatography using $60 / 40$ toluene/EtOAc to elute a small amount of starting material, followed by 60/30/10 EtOAc/toluene/ $\mathrm{MeOH}$ to elute the product, providing $0.65 \mathrm{~g}$ ( $66 \%$ yield) of $\mathbf{2 3}$ as a yellow solid. $\quad$ IR ( $\mathrm{KBr}$ pellet): $\quad 3316,1767,1716,1678,1539,1506 . \quad{ }^{1} \mathrm{H} \quad \mathrm{NMR}(2 / 1$ $\left.\mathrm{CDCl}_{3} / \mathrm{CD}_{3} \mathrm{OD}\right): \delta 1.14(\mathrm{~d}, 6 \mathrm{H}, J=6.8), 2.20-2.33(\mathrm{~m}, 5 \mathrm{H}), 3.60(\mathrm{t}, 2 \mathrm{H}, J=6.7), 3.69(\mathrm{t}$, $2 \mathrm{H}, J=6.4), 4.07-4.12(\mathrm{~m}, 4 \mathrm{H}), 4.52(\mathrm{t}, 2 \mathrm{H}, J=5.4), 6.69(\mathrm{~s}, 1 \mathrm{H}), 7.37(\mathrm{t}, 1 \mathrm{H}, J=7.9)$, 
$7.46(\mathrm{~d}, 1 \mathrm{H}, J=5.9), 7.56-7.65(\mathrm{~m}, 4 \mathrm{H}), 7.67(\mathrm{~s}, 1 \mathrm{H}), 7.80(\mathrm{~s}, 1 \mathrm{H}), 7.85(\mathrm{~d}, 1 \mathrm{H}, J=8.3)$, $7.95(\mathrm{~d}, 1 \mathrm{H}, J=8.3), 8.40(\mathrm{~d}, 1 \mathrm{H}, J=8.3), 8.77(\mathrm{~d}, 1 \mathrm{H}, J=7.3), 8.85(\mathrm{~d}, 1 \mathrm{H}, J=7.6)$, $11.92(\mathrm{~s}, 1 \mathrm{H}), 12.0(\mathrm{~s}, 1 \mathrm{H}) .{ }^{13} \mathrm{C} \mathrm{NMR}\left(2 / 1 \mathrm{CDCl}_{3} / \mathrm{CD}_{3} \mathrm{OD}\right): \delta 19.4,28.4,28.6,28.7,37.3$, $37.4,66.9,67.4,75.8,99.4,99.9,100.5,116.56(\mathrm{q}, J=287), 116.62,117.5,118.8,122.2$, $122.7,123.8,125.4,126.1,127.8,127.9,129.0,134.4,135.2,139.2,139.3,139.7,145.7$, $146.0,150.9,154.7,158.6(\mathrm{q}, J=37), 158.7(\mathrm{q}, J=37), 162.8,163.3,163.5,163.8,164.2$, 165.4. MS calcd $[\mathrm{M}+\mathrm{H}]^{+}\left(\mathrm{C}_{44} \mathrm{H}_{39} \mathrm{~N}_{8} \mathrm{O}_{11} \mathrm{~F}_{6}\right)$ : 969.2642. Found: (TOF MS ES+) 969.2615.

Pentamer 24. The same procedure as for the preparation of $\mathbf{5}$ was followed. The product was purified by silica gel chromatography using a gradient from 70/30 to 60/40 toluene/EtOAc to provide $0.34 \mathrm{~g}$ ( $75 \%$ yield) of $\mathbf{2 4}$ as a yellow solid. IR $\left(\mathrm{CHCl}_{3}\right.$ solution): 1726, 1684, 1545. ${ }^{1} \mathrm{H}$ NMR $\left(\mathrm{CDCl}_{3}\right): \delta 1.21-1.32(\mathrm{~m}, 12 \mathrm{H}), 2.30-2.36(\mathrm{~m}, 5 \mathrm{H}), 2.49-2.53$ (m, 3H), $3.19(\mathrm{~s}, 3 \mathrm{H}), 3.66-3.76(\mathrm{~m}, 3 \mathrm{H}), 3.83-3.94(\mathrm{~m}, 5 \mathrm{H}), 4.09-4.14(\mathrm{~m}, 2 \mathrm{H}), 4.19-4.23$ (m, 2H), 4.27-4.29 (m, 1H), $4.40(\mathrm{t}, 1 \mathrm{H}, J=7.2), 4.62-4.65(\mathrm{~m}, 1 \mathrm{H}), 4.77-4.78(\mathrm{~m}, 1 \mathrm{H})$, $6.50(\mathrm{~s}, 1 \mathrm{H}), 6.67(\mathrm{~s}, 1 \mathrm{H}), 6.76(\mathrm{~s}, 1 \mathrm{H}), 7.06(\mathrm{br} \mathrm{s}, 1 \mathrm{H}), 7.26-7.35(\mathrm{~m}, 4 \mathrm{H}), 7.48(\mathrm{~s}, 1 \mathrm{H})$, $7.52(\mathrm{~s}, 1 \mathrm{H}), 7.59(\mathrm{br} \mathrm{s}, 1 \mathrm{H}), 7.64-7.69(\mathrm{~m}, 3 \mathrm{H}), 7.85(\mathrm{~d}, 1 \mathrm{H}, J=8.3), 7.98(\mathrm{~d}, 1 \mathrm{H}, J=8.3)$, $8.01(\mathrm{~d}, 1 \mathrm{H}, J=7.6), 8.07$ (t, 2H, $J=8.9), 8.17(\mathrm{br} \mathrm{s}, 1 \mathrm{H}), 8.39$ (d, 1H, $J=7.8), 8.46$ (d, $1 \mathrm{H}, J=7.3), 8.55(\mathrm{~d}, 1 \mathrm{H}, J=7.9), 11.57(\mathrm{~s}, 1 \mathrm{H}), 11.67(\mathrm{~s}, 1 \mathrm{H}), 11.74(\mathrm{~s}, 1 \mathrm{H}), 12.05(\mathrm{~s}, 1 \mathrm{H})$. ${ }^{13} \mathrm{C}$ NMR $\left(\mathrm{CDCl}_{3}\right): \delta 19.5,19.56,19.59,19.64,28.0,28.4,28.5,28.6,37.2,37.67,37.72$, $52.3,66.2,66.7,67.1,75.5,75.8,97.0,98.1,99.6,100.2,100.5,114.9,116.08,116.11$ (q, $J$ $=288), 116.3(\mathrm{q}, J=288), 116.4,116.5,116.6,116.8,117.3,118.0,121.4,121.7,122.2$, $122.5,123.5,124.8,125.7,126.79,126.83,127.1,127.9,128.7,133.1,133.4,134.3,134.6$, $138.1,138.4,138.5,138.9,139.1,144.7,145.6,148.7,149.2,150.5,154.0,157.8(\mathrm{q}, J=$ 
37), $157.96(\mathrm{q}, J=37), 158.00(\mathrm{q}, J=37), 160.5,160.9,161.5,161.7,161.8,162.8,162.9$, 163.4, 164.0, 164.9. MS calcd $[\mathrm{M}+\mathrm{H}]^{+}\left(\mathrm{C}_{74} \mathrm{H}_{67} \mathrm{~N}_{13} \mathrm{O}_{16} \mathrm{~F}_{9}\right)$ : 1564.4685. Found: (TOF MS ES+) 1564.4652.

Pentamer amine 25. The same procedure as for the preparation of $\mathbf{3}$ was followed to provide $120 \mathrm{mg}$ (99\% yield) of $\mathbf{2 5}$ as a yellow solid. IR (thin film): 3305, 1716, 1678, 1538. ${ }^{1} \mathrm{H}$ NMR $\left(\mathrm{CDCl}_{3}\right): \delta 1.21-1.33(\mathrm{~m}, 12 \mathrm{H}), 2.28-2.36(\mathrm{~m}, 5 \mathrm{H}), 2.43-2.58(\mathrm{~m}, 3 \mathrm{H})$, $3.21(\mathrm{~s}, 3 \mathrm{H}), 3.69-3.92(\mathrm{~m}, 8 \mathrm{H}), 4.00-4.12(\mathrm{~m}, 2 \mathrm{H}), 4.20-4.27(\mathrm{~m}, 2 \mathrm{H}), 4.30-4.36(\mathrm{~m}, 1 \mathrm{H})$, 4.40-4.46 (m, 1H), 4.58-4.63 (m, 1H), 4.66-4.74 (m, 1H), $5.72(\mathrm{~d}, 1 \mathrm{H}, J=7.6), 6.52(\mathrm{~s}$, 1H), $6.69(\mathrm{~s}, 1 \mathrm{H}), 6.75(\mathrm{~s}, 1 \mathrm{H}), 6.91(\mathrm{t}, 1 \mathrm{H}, J=7.8), 6.99(\mathrm{br} \mathrm{s}, 1 \mathrm{H}), 7.26-7.31(\mathrm{~m}, 2 \mathrm{H})$, 7.35-7.37 (m, 2H), 7.44-7.47 (m, 2H), 7.66-7.72 (m, 3H), $7.86(\mathrm{~d}, 1 \mathrm{H}, J=8.3), 7.92-7.95$ $(\mathrm{m}, 2 \mathrm{H}), 7.97(\mathrm{~d}, 1 \mathrm{H}, J=7.6), 8.06(\mathrm{~d}, 1 \mathrm{H}, J=8.3), 8.37(\mathrm{t}, 1 \mathrm{H}, J=5.5), 8.47(\mathrm{~d}, 1 \mathrm{H}, J=$ 7.6), $8.61(\mathrm{~d}, 1 \mathrm{H}, J=7.6), 11.74(\mathrm{~s}, 1 \mathrm{H}), 11.77(\mathrm{~s}, 1 \mathrm{H}), 11.89(\mathrm{~s}, 1 \mathrm{H}), 12.09(\mathrm{~s}, 1 \mathrm{H}) .{ }^{13} \mathrm{C}$ $\operatorname{NMR}\left(\mathrm{CDCl}_{3}\right): \delta 19.4,19.5,19.57,19.62,27.9,28.3,28.4,28.5,29.9,37.4,37.5,37.6$, 52.3, 66.3, 66.6, 75.3, 75.7, 97.1, 97.9, 98.9, 99.3, 100.2, 109.0, 109.6, 115.3, 115.4, 115.7, $115.9,116.0,116.1(\mathrm{q}, J=288), 116.3(\mathrm{q}, J=288), 116.7,117.1,117.6,121.3,121.6$, $122.1,122.3,122.6,127.1,127.2,127.3,128.5,133.1,133.4,133.9,134.3,136.3,137.8$, $138.3,139.0,143.3,144.9,148.77,148.81,149.0,150.0,157.85(\mathrm{q}, J=37), 157.92(\mathrm{q}, J=$ 37), $158.06(\mathrm{q}, J=37), 161.0,161.1,161.5,161.7,161.8,162.6,162.7,163.3,164.1,164.7$. MS calcd $[\mathrm{M}+\mathrm{H}]^{+}\left(\mathrm{C}_{74} \mathrm{H}_{69} \mathrm{~N}_{13} \mathrm{O}_{14} \mathrm{~F}_{9}\right)$ : 1534.4943. Found: (TOF MS ES+) 1534.4951.

Heptamer 26. The same procedure as for the preparation of $\mathbf{1 2}$ was followed to provide $165 \mathrm{mg}$ (78 \% yield) of $\mathbf{2 6}$ as a yellow solid. IR (thin film): 3310, 1716, 1678, 1534. ${ }^{1} \mathrm{H}$ 
NMR $\left(\mathrm{CDCl}_{3}\right) \delta 1.17-1.23(\mathrm{~m}, 18 \mathrm{H}), 2.12-2.58(\mathrm{~m}, 11 \mathrm{H}), 3.06(\mathrm{~s}, 3 \mathrm{H}), 3.48-3.62(\mathrm{~m}, 2 \mathrm{H})$, 3.77-3.82 (m, 4H), 3.93-4.22 (m, 11H), 4.34-4.41 (m, 1H), 4.46-4.57 (m, 3H), 4.59-4.65 (m, 1H), $6.41(\mathrm{~s}, 1 \mathrm{H}), 6.45(\mathrm{~s}, 1 \mathrm{H}), 6.56(\mathrm{~s}, 1 \mathrm{H}), 6.58(\mathrm{~s}, 1 \mathrm{H}), 6.68(\mathrm{~s}, 1 \mathrm{H}), 6.95(\mathrm{br} \mathrm{s}, 1 \mathrm{H})$, 7.18-7.31 (m, 6H), 7.35-7.40 (m, 3H), 7.42-7.49 (m, 3H), 7.52 (br s, 1H), $7.65(\mathrm{~d}, 1 \mathrm{H}, J=$ 8.3), 7.76-7.78 (m, 2H), $7.88(\mathrm{t}, 2 \mathrm{H}, J=9.4), 8.01(\mathrm{br} \mathrm{s}, 1 \mathrm{H}), 8.04-8.12(\mathrm{~m}, 3 \mathrm{H}), 8.23(\mathrm{~d}$, $1 \mathrm{H}, J=7.3), 8.28(\mathrm{~d}, 1 \mathrm{H}, J=7.6), 8.37-8.42(\mathrm{~m}, 2 \mathrm{H}), 11.19(\mathrm{~s}, 2 \mathrm{H}), 11.32(\mathrm{~s}, 1 \mathrm{H}), 11.3(\mathrm{~s}$, $1 \mathrm{H}), 11.40(\mathrm{~s}, 1 \mathrm{H}), 11.80(\mathrm{~s}, 1 \mathrm{H}) .{ }^{13} \mathrm{C} \mathrm{NMR}\left(\mathrm{CDCl}_{3}\right): \delta 19.3,19.4,19.5,19.7,28.0,28.2$, $28.3,28.35,28.43,28.8,29.9,37.0,37.4,39.6,52.1,65.7,66.3,66.4,68.6,75.2,75.6,75.8$, 97.0, 97.8, 98.4, 98.5, 99.3, 100.0, 100.1, 115.5, 115.8, 116.13, $116.13(\mathrm{q}, J=288), 116.19$ $(\mathrm{q}, J=288), 116.23,116.23(\mathrm{q}, J=288), 116.37,116.47,116.50(\mathrm{q}, J=288), 117.0,117.2$, $117.3,117.56,117.62,117.7,117.9,121.4,121.8,122.09,122.13,122.2,123.8,124.4$, $125.9,126.2,126.7,126.8,126.9,127.3,128.3,132.4,132.6,132.8,133.6,133.95,134.04$, $137.5,137.6,137.9,138.1,138.5,138.7,138.9,144.6,144.9,148.5,148.6,148.8,149.3$, $150.1,153.1,157.6(\mathrm{q}, J=37), 157.9(\mathrm{q}, J=36), 158.0(\mathrm{q}, J=36), 158.1(\mathrm{q}, J=36), 159.8$, $160.0,160.2,160.5,161.0,161.2,161.5,162.66,162.71,162.9,163.0,163.1,163.5,164.5$. MS calcd $[\mathrm{M}+\mathrm{H}]^{+}\left(\mathrm{C}_{103} \mathrm{H}_{93} \mathrm{~N}_{18} \mathrm{O}_{21} \mathrm{~F}_{12}\right): 2145.6571$. Found: (TOF MS ES+) 2145.6560.

Decamer 28. The heptamer 26 was reduced to the amine 27 using the same procedure as for the preparation of $\mathbf{3}$ and was taken to the next step without further purification. The decamer $\mathbf{2 8}$ was then prepared by the same procedure as for the preparation of $\mathbf{5}$. The product was purified by silica gel chromatography on a chromatotron using 75/25 EtOAc/hexanes as the eluent to provide $30 \mathrm{mg}$ (45\% yield) IR (thin film): 3313,1715 , 1670, 1537. ${ }^{1} \mathrm{H}$ NMR $\left(\mathrm{CDCl}_{3}\right): \delta$ 1.13-1.36 (m, 24H), 2.17-2.48 (m, 16H), 2.93 (s, 3H), 
3.54-4.21 (m, 26H), 4.26-4.34 (m, 3H), 4.38-4.44 (m, 3H), $5.99(\mathrm{~s}, 1 \mathrm{H}), 6.14(\mathrm{~s}, 2 \mathrm{H}), 6.26$ (s, 1H), $6.28(\mathrm{~s}, 1 \mathrm{H}), 6.38(\mathrm{~s}, 1 \mathrm{H}), 6.41(\mathrm{~s}, 1 \mathrm{H}), 6.45(\mathrm{~s}, 1 \mathrm{H}), 6.93-7.05(\mathrm{~m}, 7 \mathrm{H}), 7.10-7.38$ $(\mathrm{m}, 18 \mathrm{H}), 7.48(\mathrm{~d}, 2 \mathrm{H}, J=7.3), 7.54(\mathrm{~d}, 2 \mathrm{H}, J=7.6), 7.60(\mathrm{~d}, 1 \mathrm{H}, J=8.1), 7.72(\mathrm{~m}, 8 \mathrm{H})$, 7.90-8.13 (m, 8H), 10.63 (s, 1H), 10.67 (s, 2H), 10.87 (s, 2H), 10.95 (s, 1H), 10.07 (s, 1H), $11.11(\mathrm{~s}, 1 \mathrm{H}), 11.44(\mathrm{~s}, 1 \mathrm{H}) .{ }^{13} \mathrm{C} \mathrm{NMR}\left(\mathrm{CDCl}_{3}\right): \delta 19.26,19.32,19.36,19.42,19.47$, $19.54,19.6,19.72,19.75,28.20,28.23,28.3,28.39,28.44,28.8,37.19,37.22,37.6,38.7$, 38.9, 39.4, 52.1, 65.9, 66.1, 66.5, 67.2, 68.0, 68.2, 75.2, 75.4, 97.0, 97.6, 97.7, 98.2, 98.6, $98.99,99.02,99.4,99.8,100.0,115.3,115.4,115.5,115.6,116.0,116.12,116.12(\mathrm{q}, J=$ $289), 116.16,116.17$ (q, $J=288), 116.22(\mathrm{q}, J=287), 116.26,116.29(\mathrm{q}, J=288), 116.32$ $(\mathrm{q}, J=288), 116.40,116.5(\mathrm{q}, J=288), 116.7,117.0,117.3,118.0,121.1,121.3,121.7$, $121.8,121.9,122.0,122.3,122.4,123.2,124.4,125.18,125.23,125.6,125.8,126.1$, $126.40,126.44,126.6,126.9,127.3,127.4,127.5,128.4,132.1,132.2,132.5,132.6,132.8$, 133.3, 133.9, 134.1, 137.2, 137.3, 137.4, 137.7, 137.9, 138.0, 138.5, 138.6, 144.4, 145.1, 148.0, 148.1, 148.3, 148.5, 148.6, 148.8, 149.3, 149.8, 153.3, 157.5-158.4 (m), 158.7, $158.8,159.5,159.6,159.8,160.0,160.4,160.8,160.9,161.4,161.9,162.1,162.2,162.4$ 162.8, 163.0, 163.18, 163.25, 164.5. MS calcd $[\mathrm{M}+\mathrm{H}]^{+}\left(\mathrm{C}_{147} \mathrm{H}_{131} \mathrm{~N}_{26} \mathrm{O}_{29} \mathrm{~F}_{18}\right): 3065.9288$. Found: (TOF MS ES+) 3065.8936. 


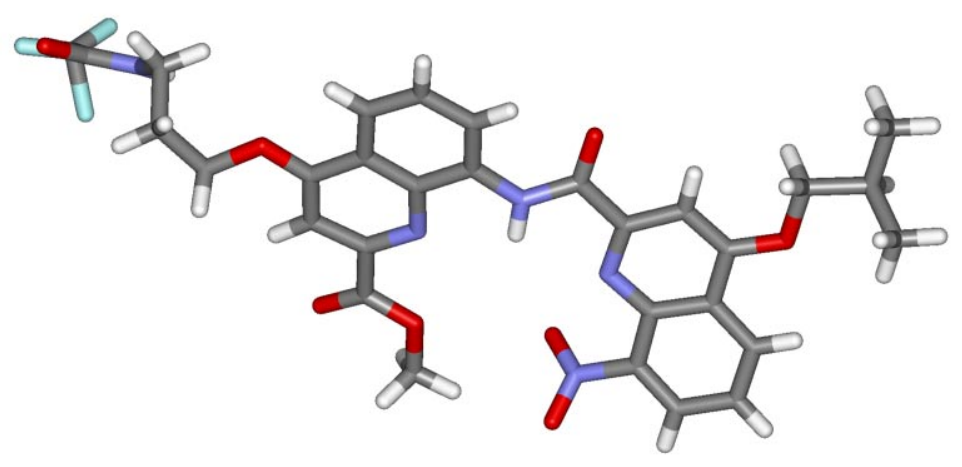

Figure 1. X-ray crystal structure of dimer 5. Included solvent molecules have been omitted for clarity.
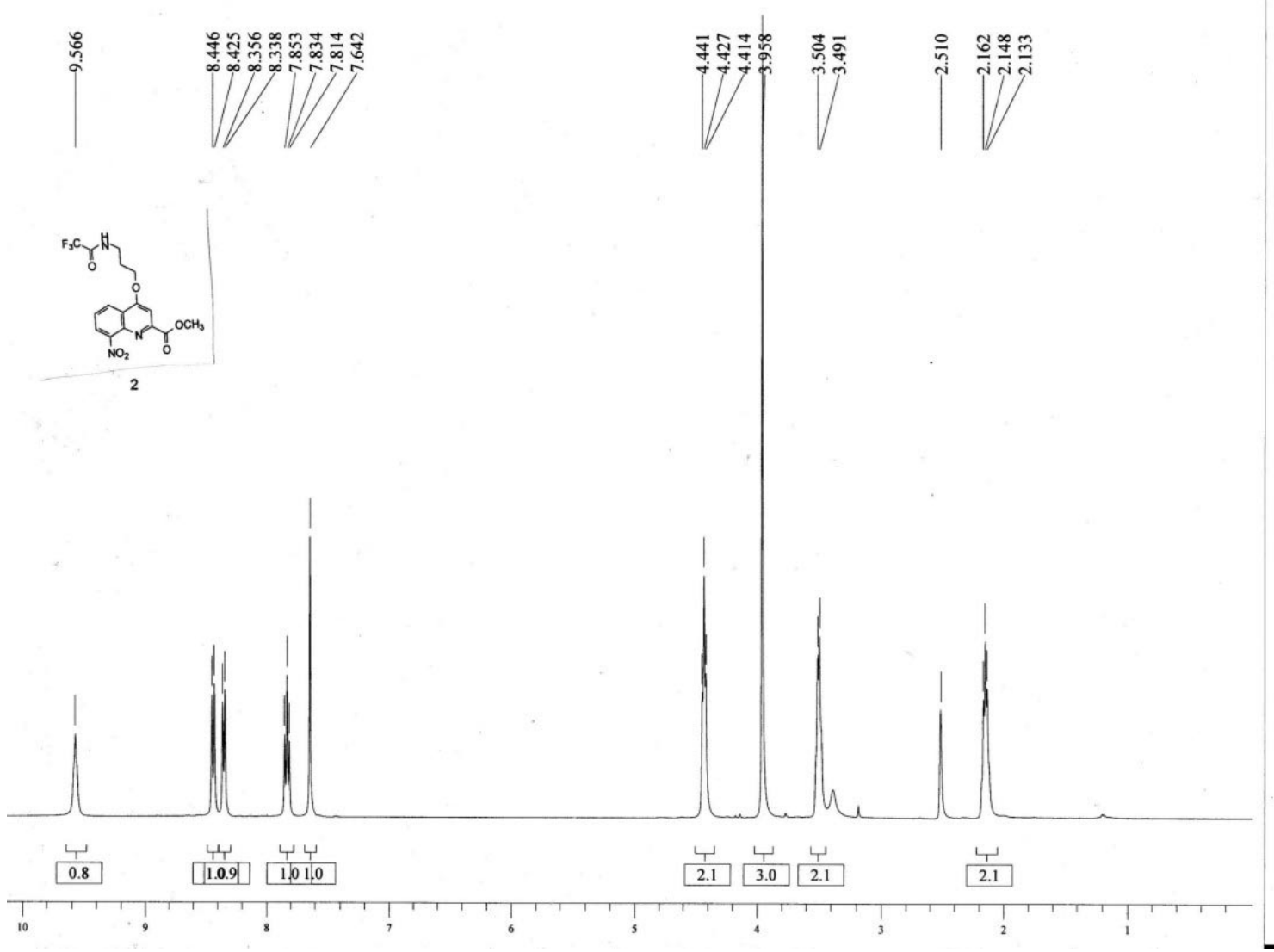

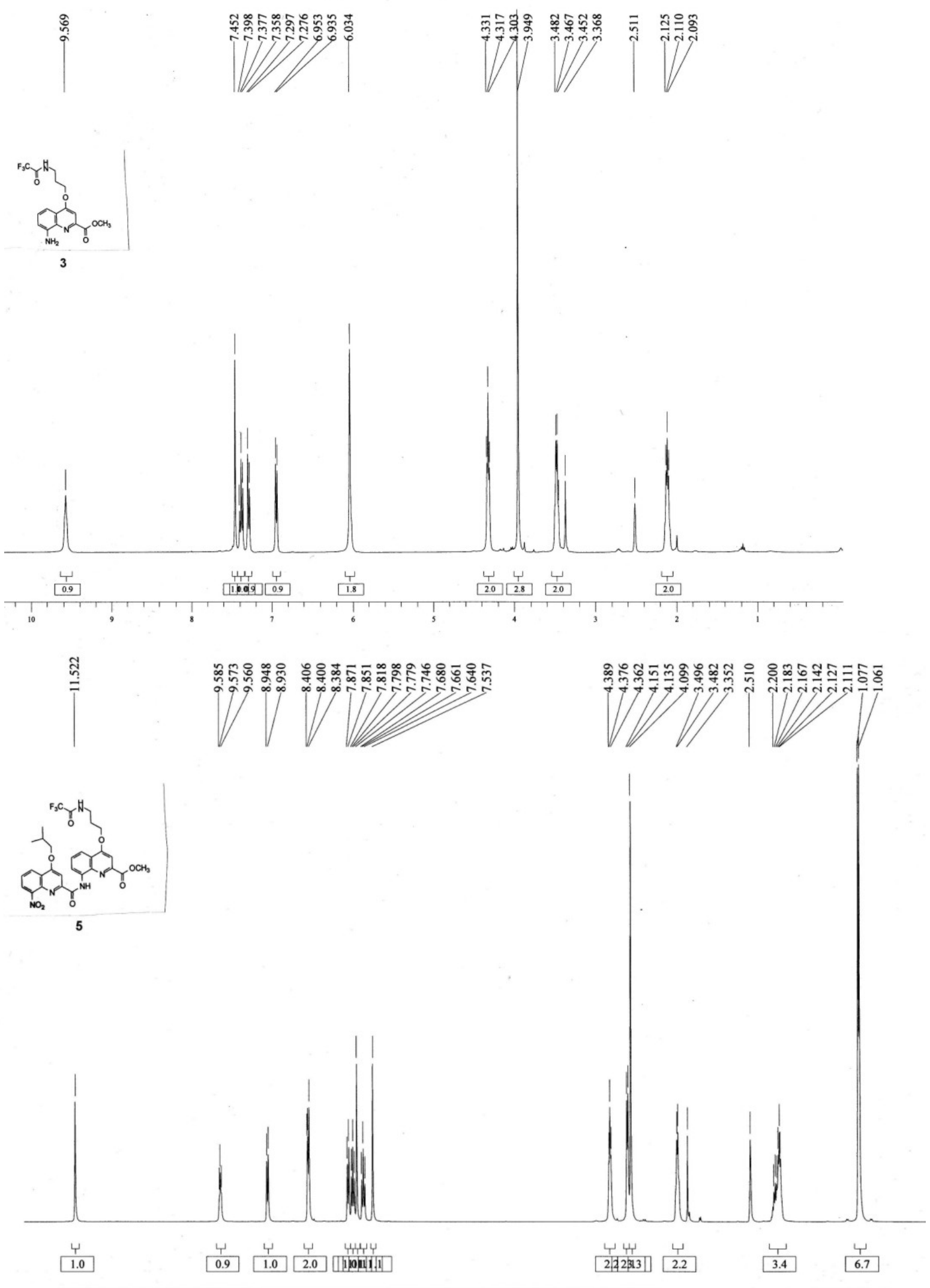

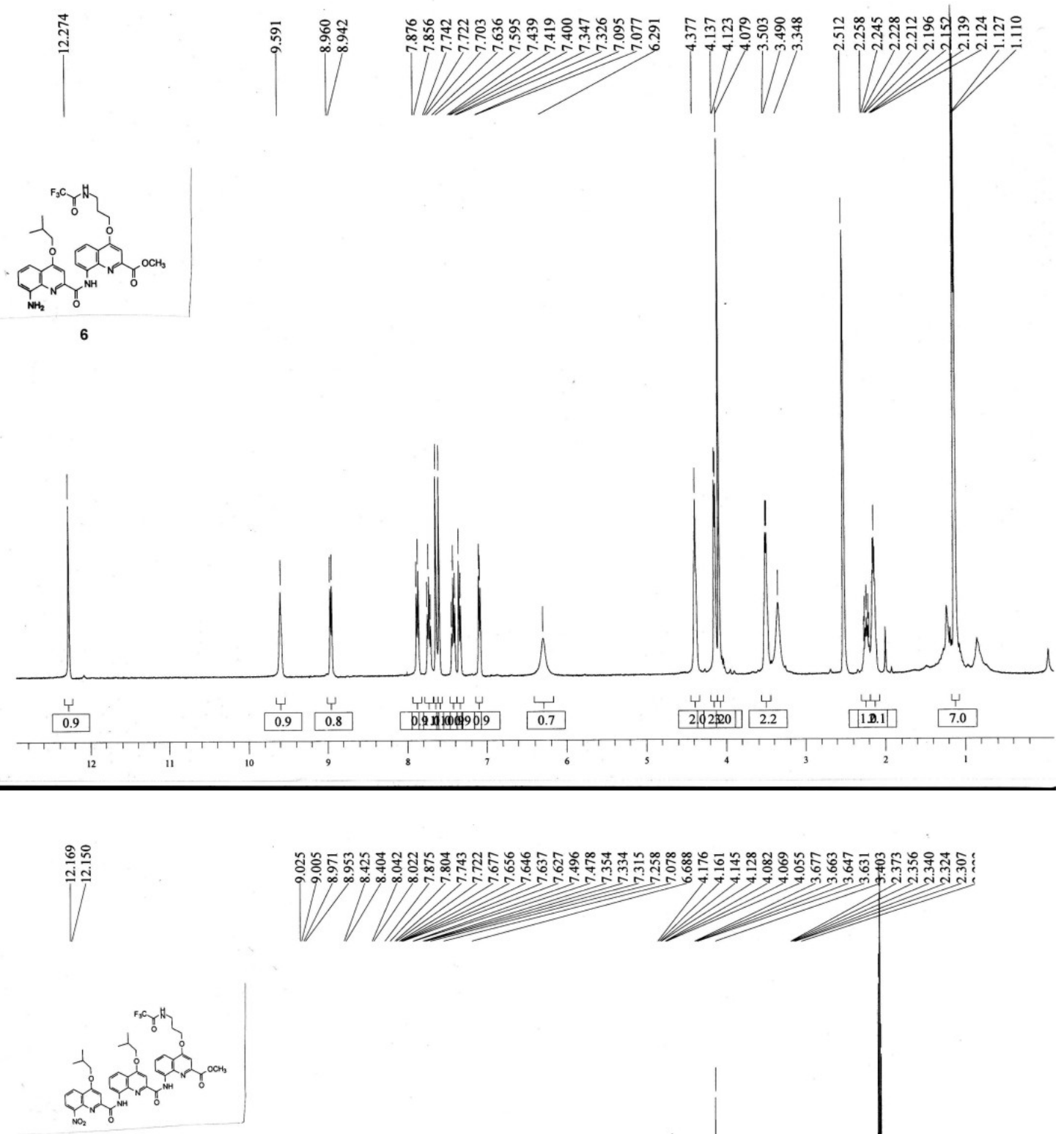

7

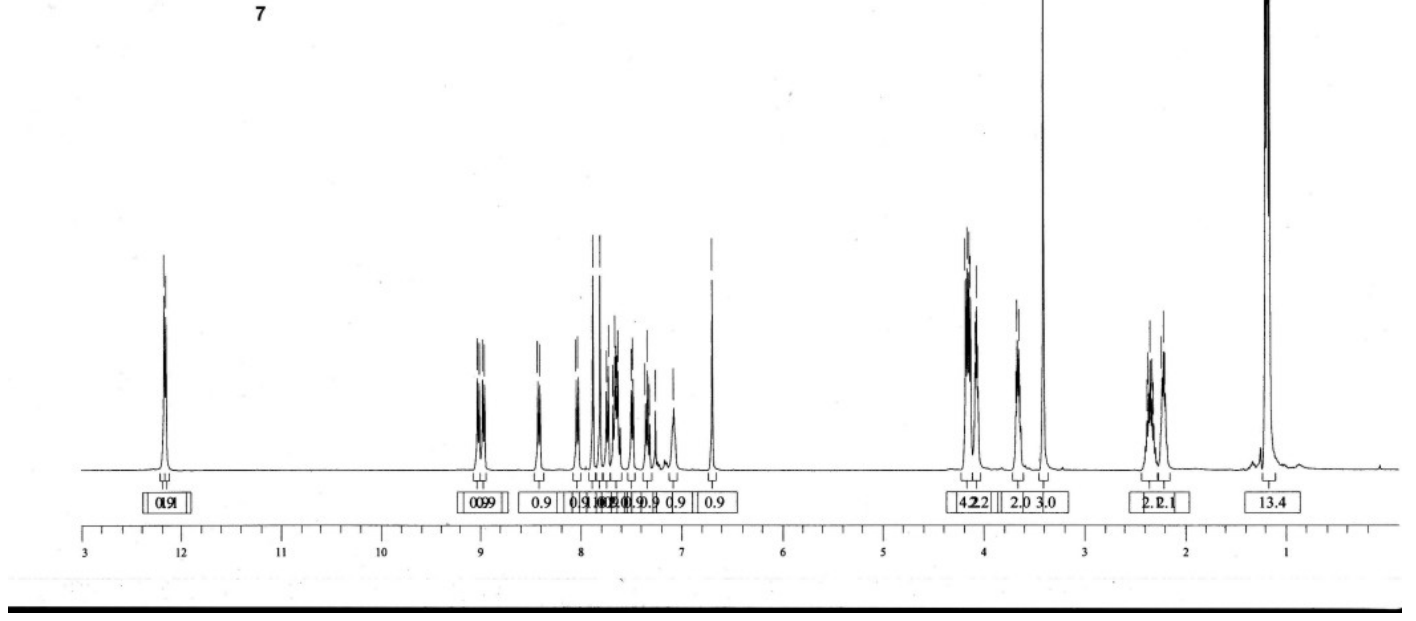



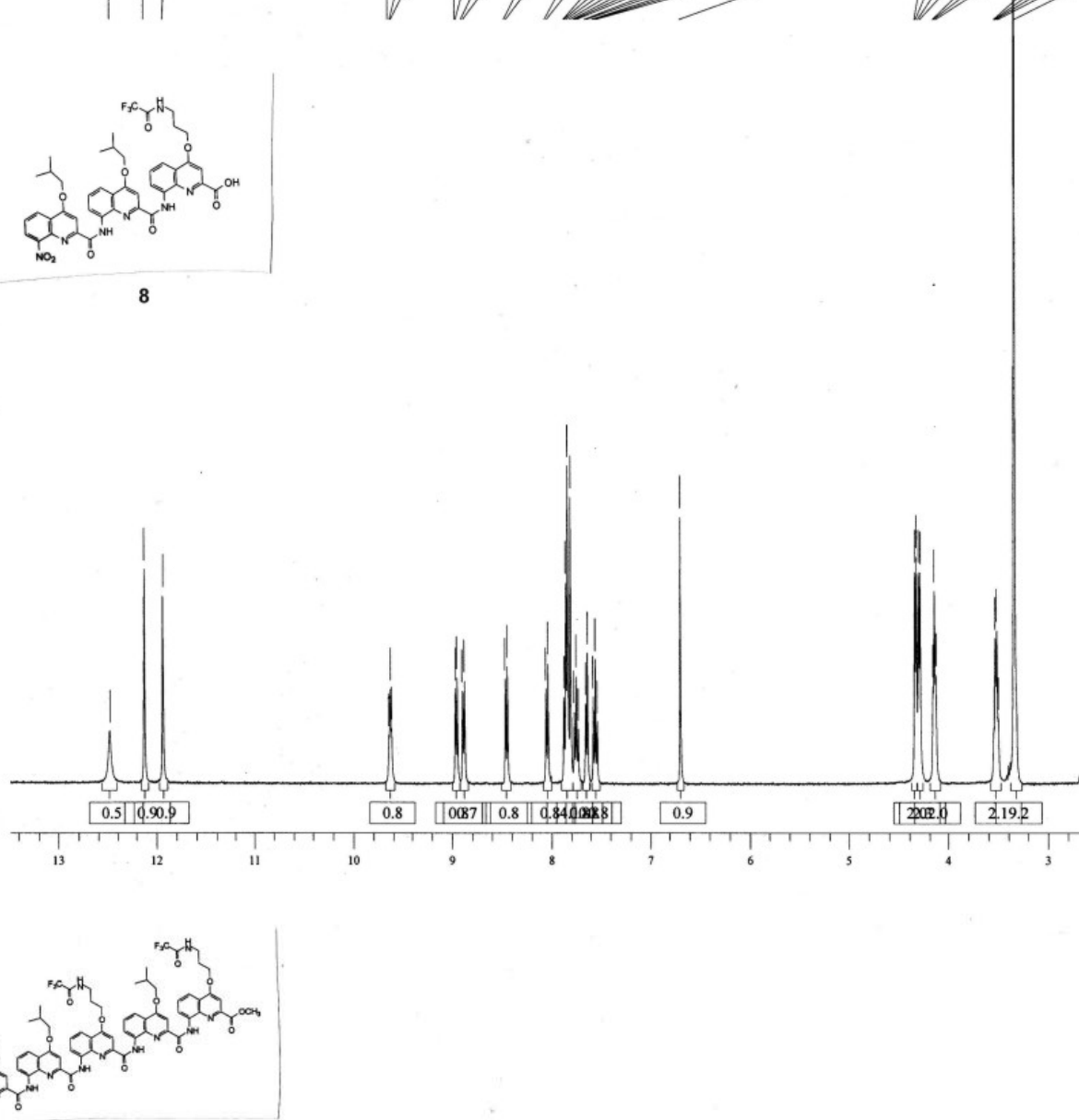

9

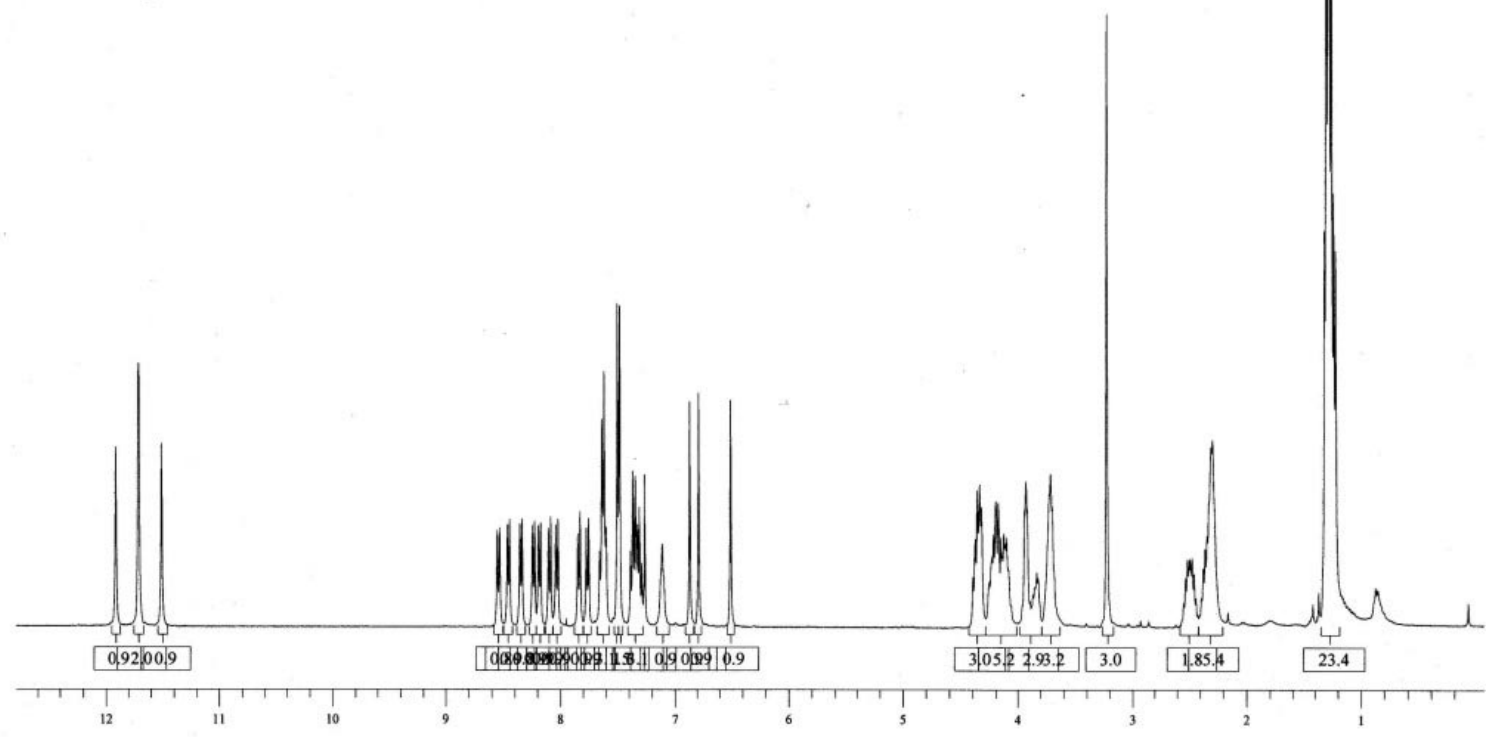



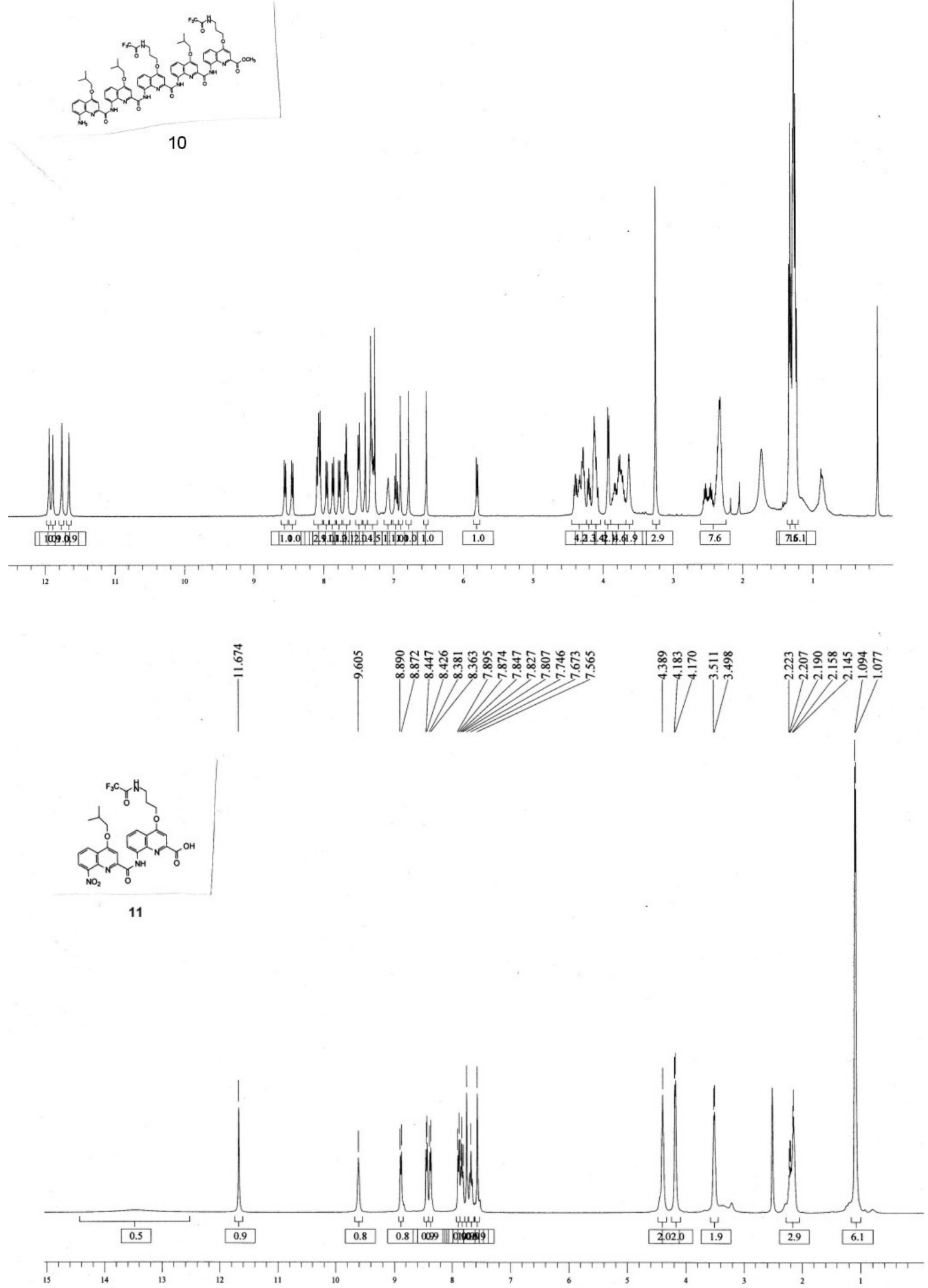

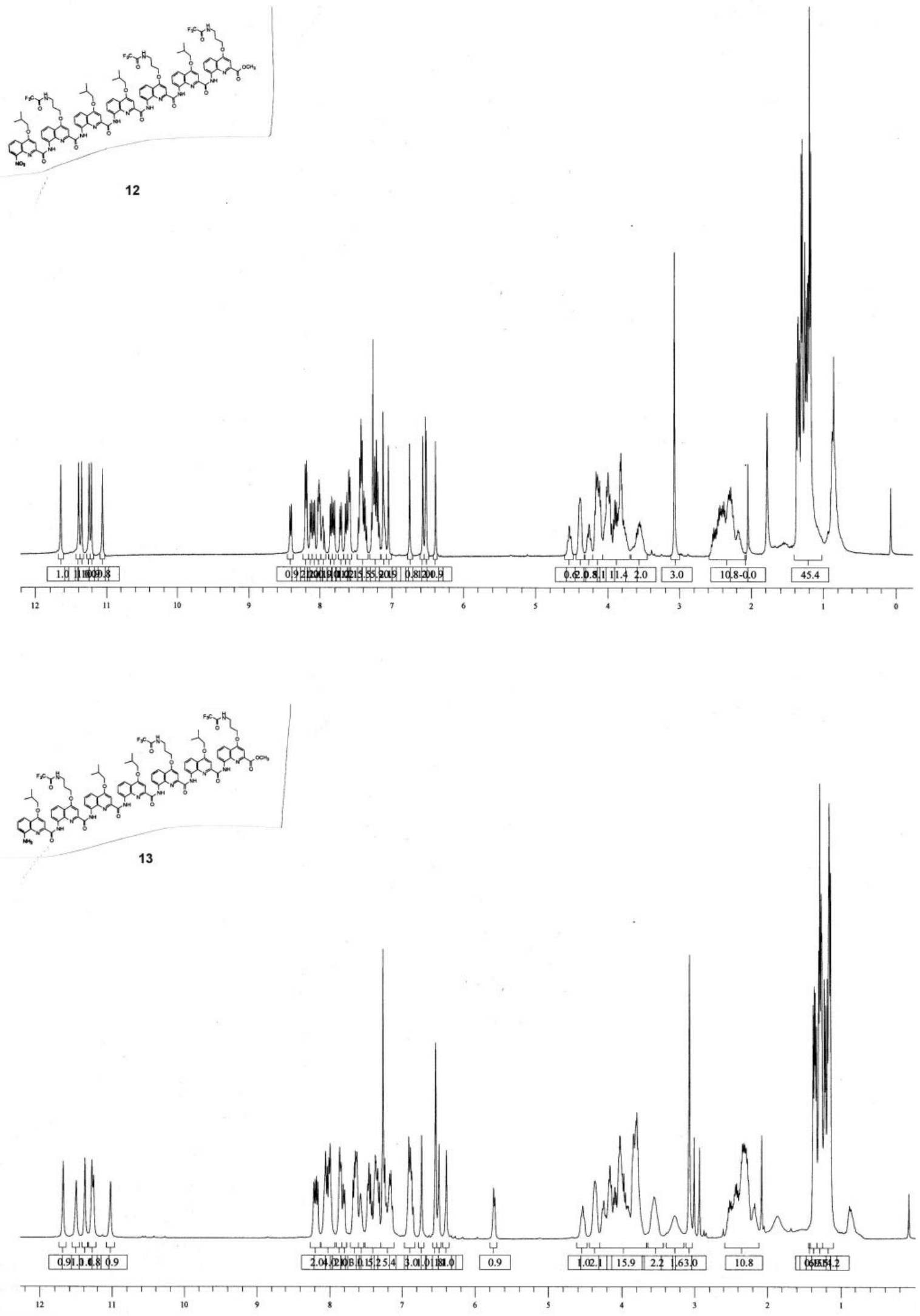

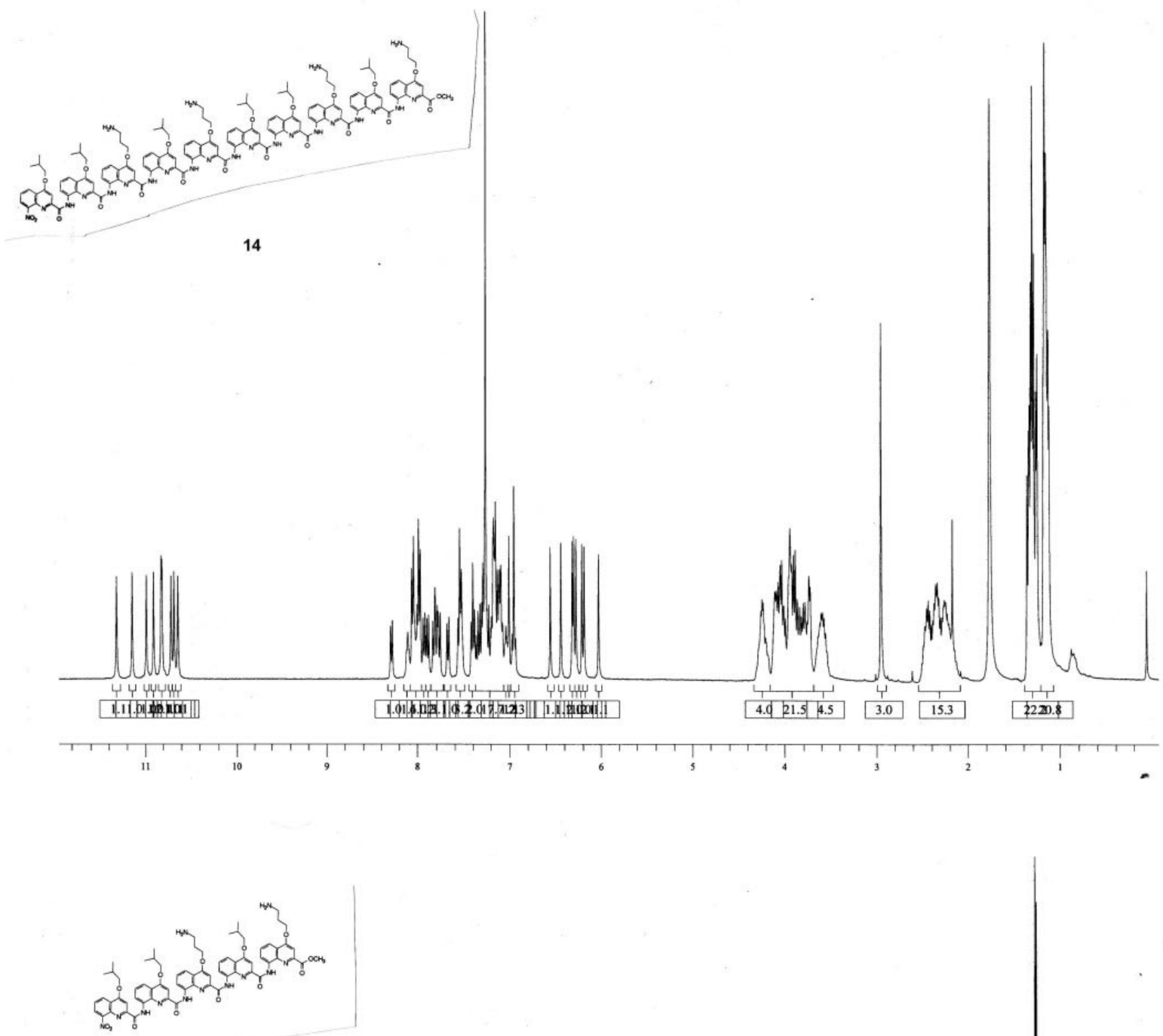

15

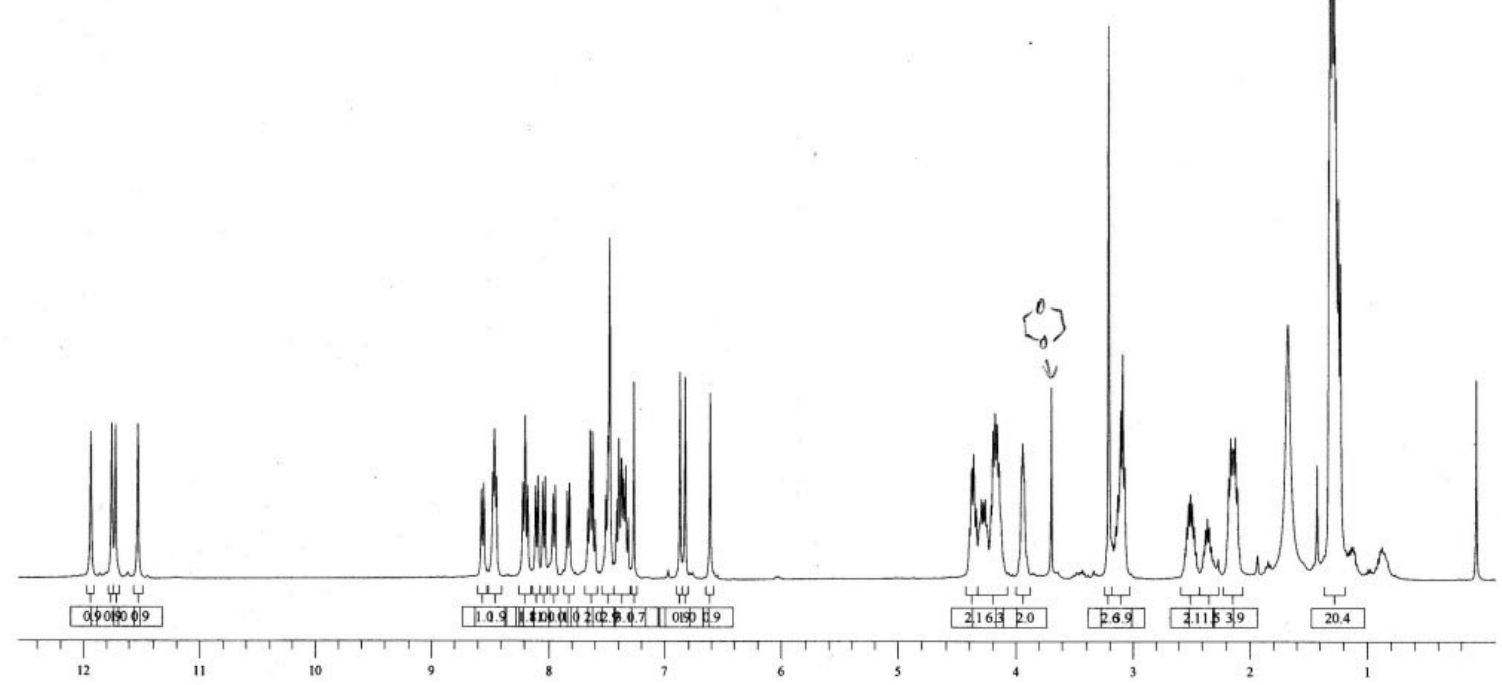



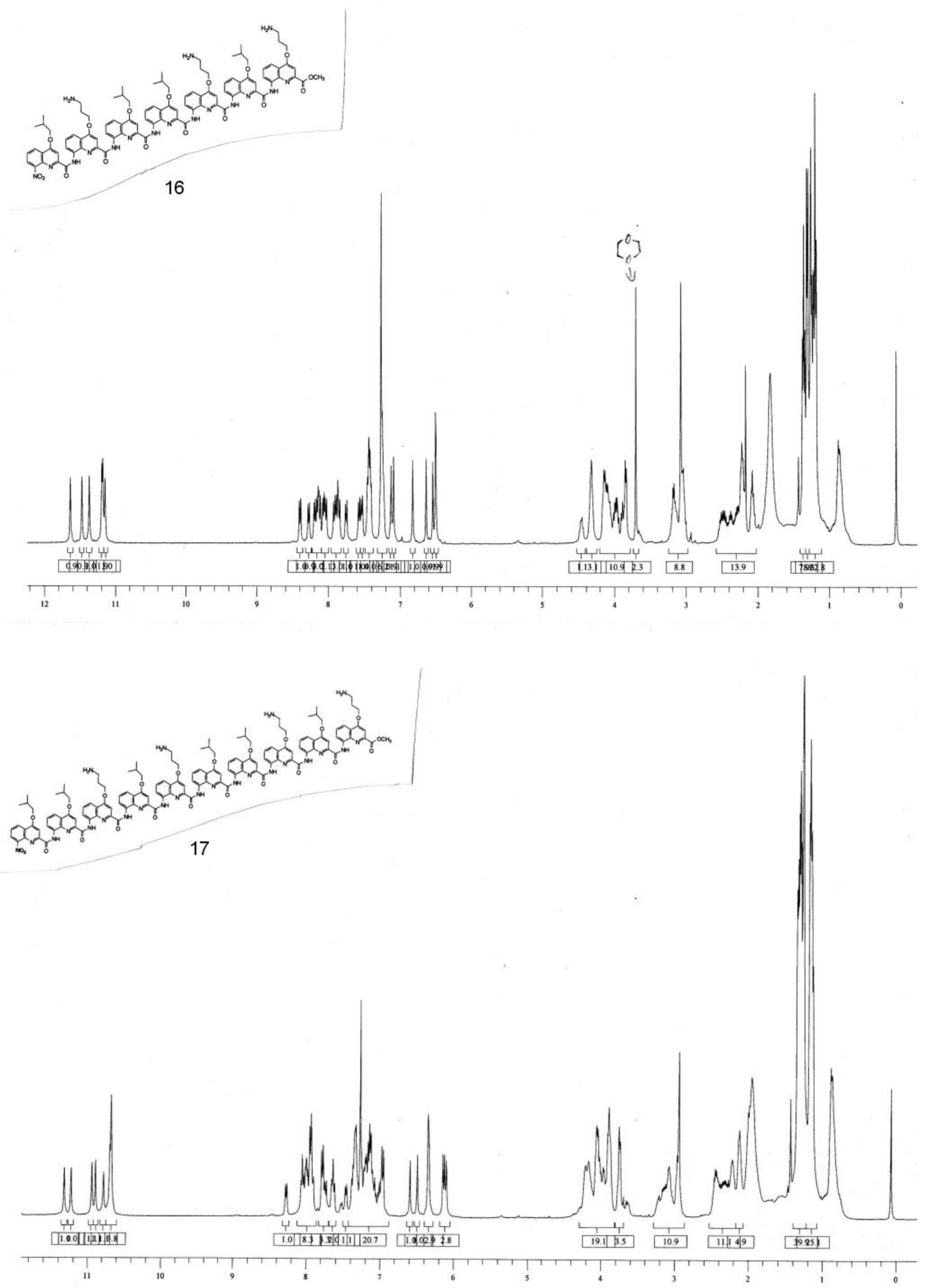

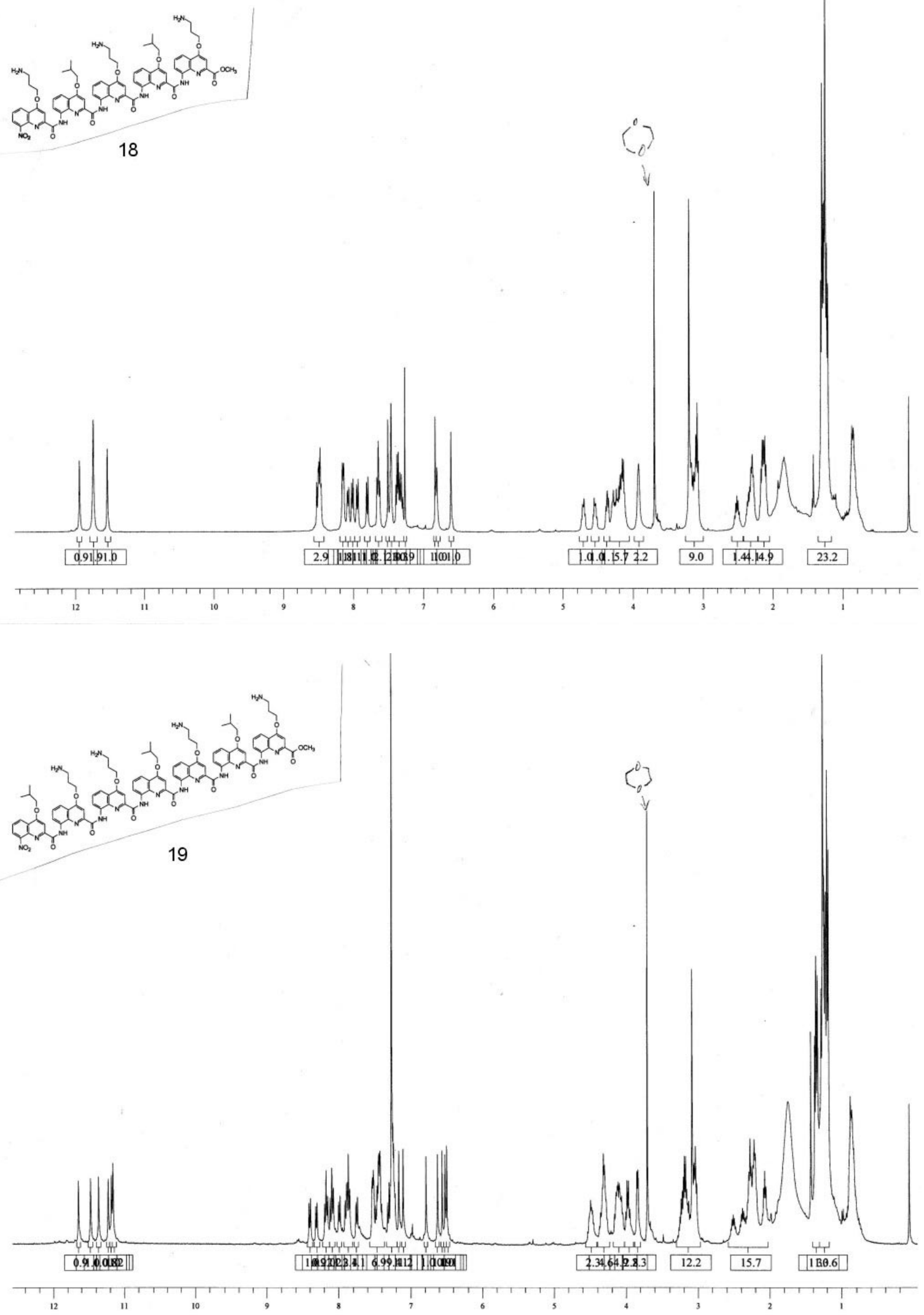

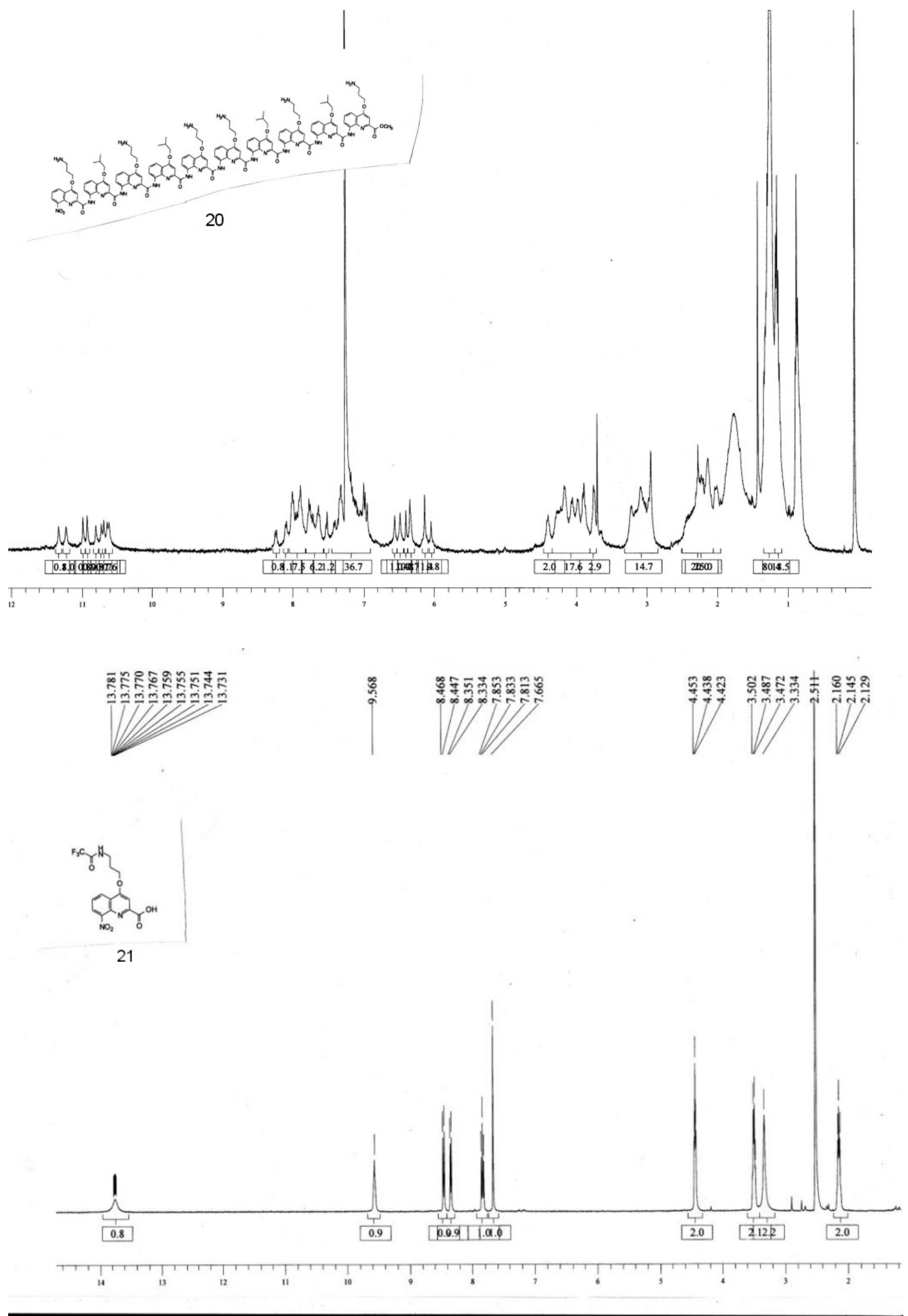

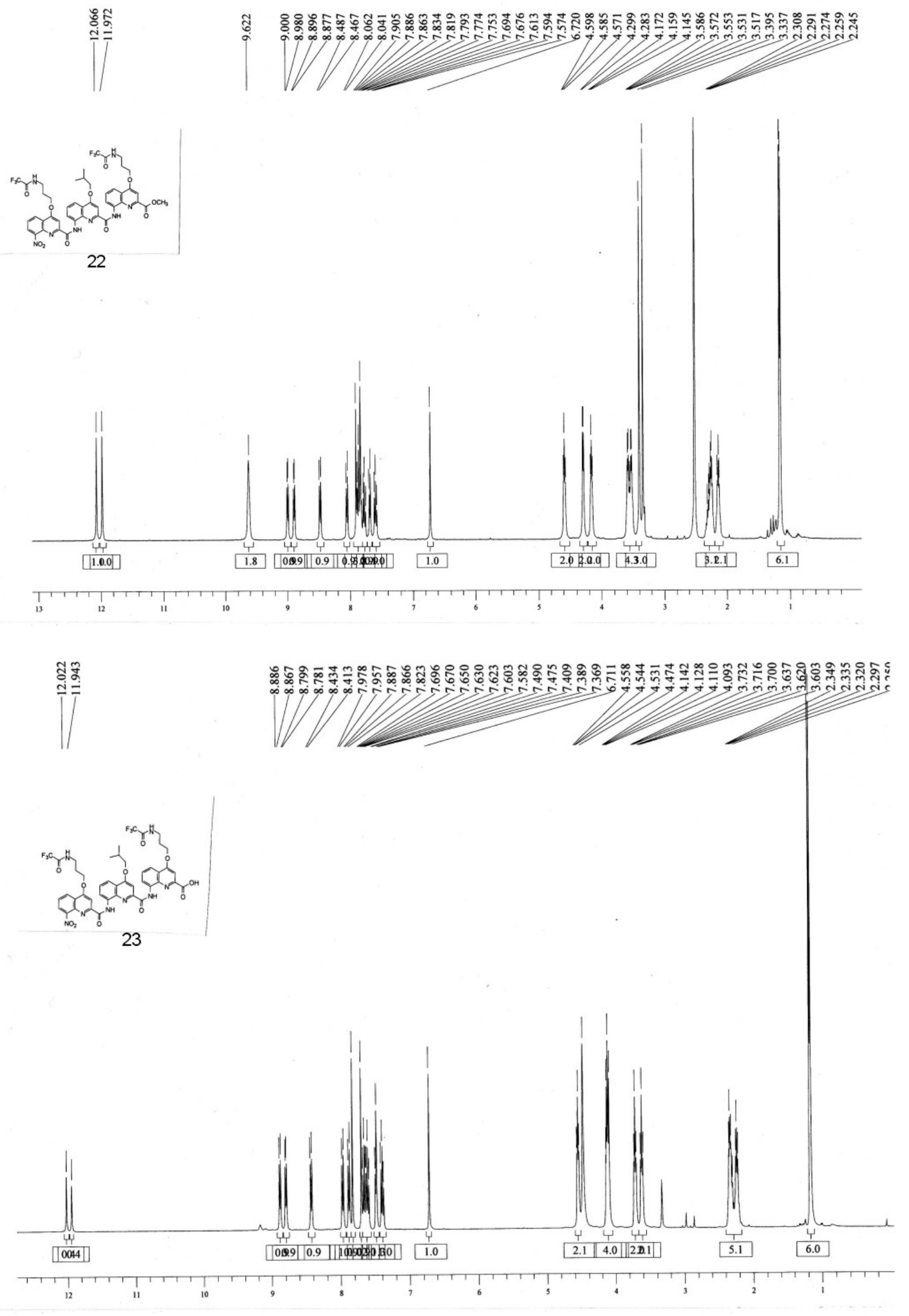

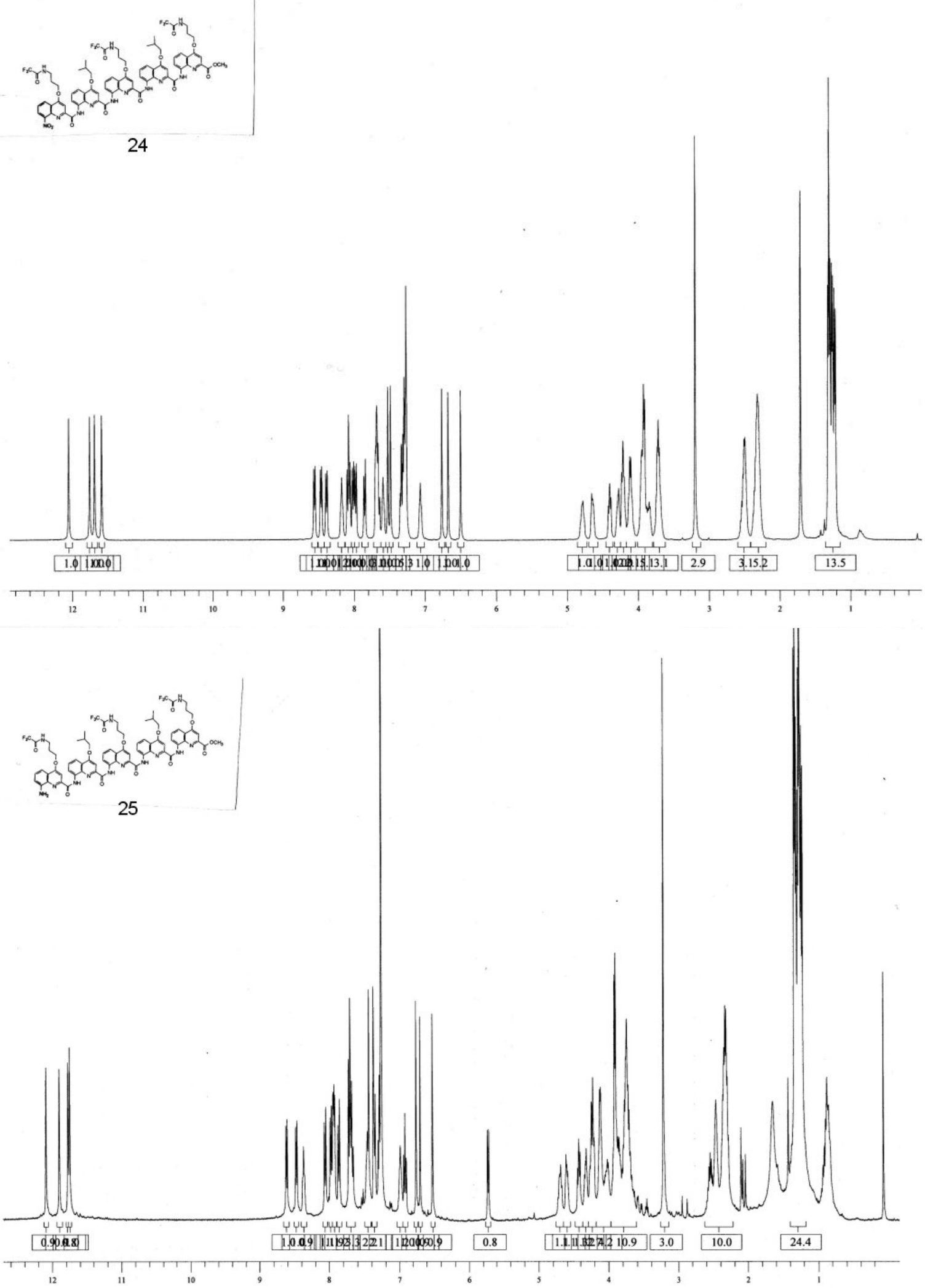

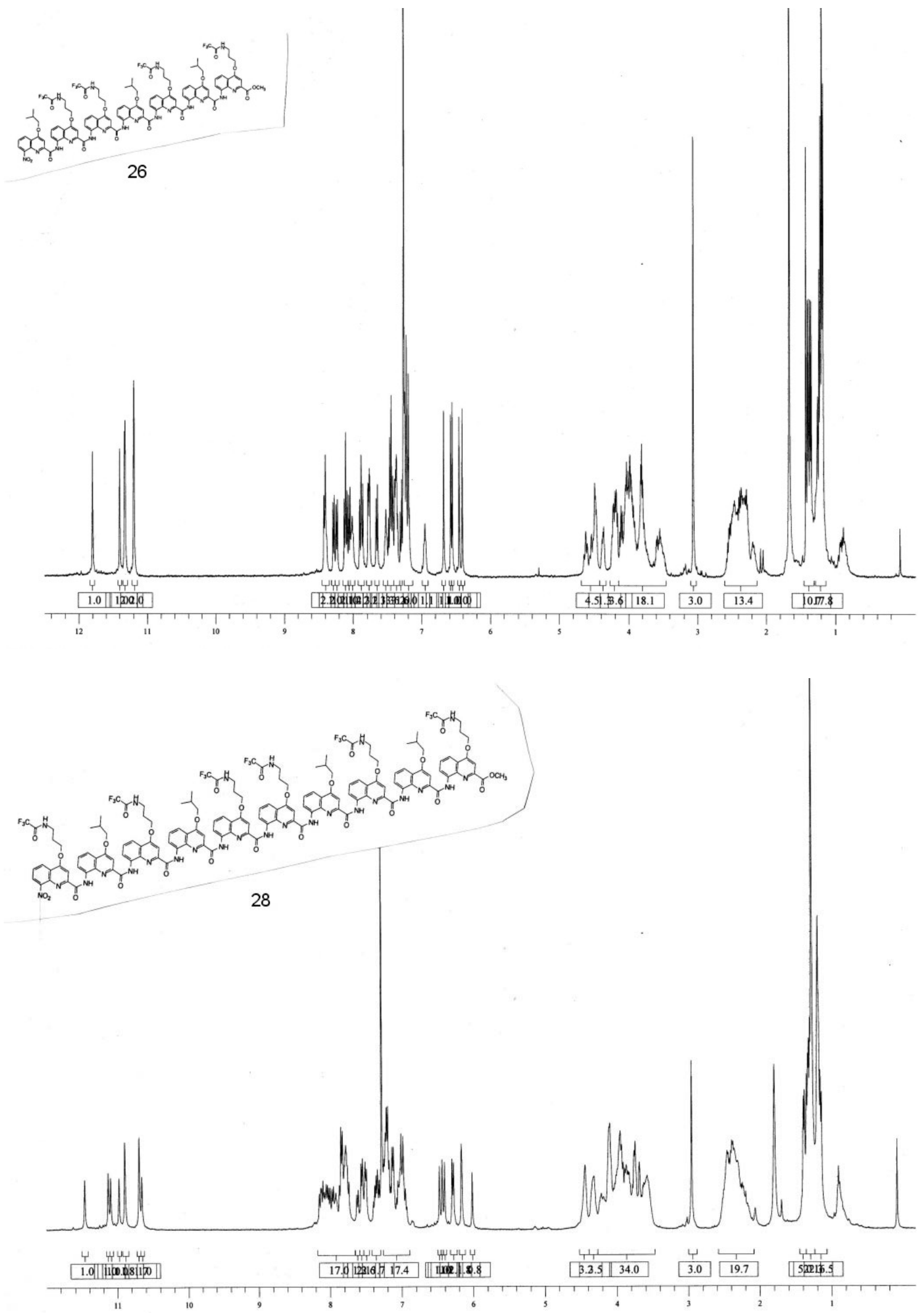